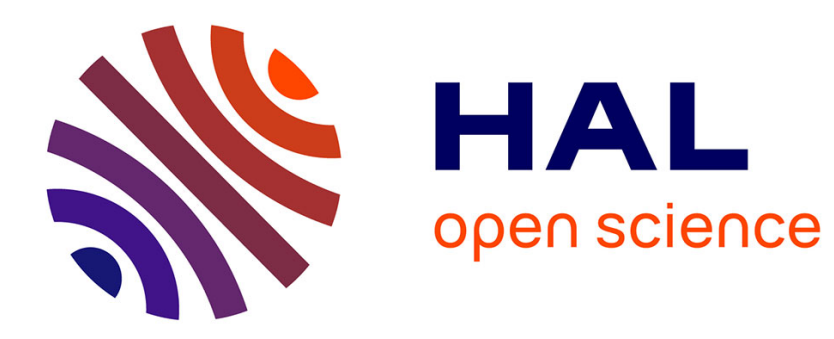

\title{
Reactive preferential structures and nonmonotonic consequence
}

\author{
Dov Gabbay, Karl Schlechta
}

\section{To cite this version:}

Dov Gabbay, Karl Schlechta. Reactive preferential structures and nonmonotonic consequence. 2008. hal-00311940

\author{
HAL Id: hal-00311940 \\ https://hal.science/hal-00311940
}

Preprint submitted on 22 Aug 2008

HAL is a multi-disciplinary open access archive for the deposit and dissemination of scientific research documents, whether they are published or not. The documents may come from teaching and research institutions in France or abroad, or from public or private research centers.
L'archive ouverte pluridisciplinaire HAL, est destinée au dépôt et à la diffusion de documents scientifiques de niveau recherche, publiés ou non, émanant des établissements d'enseignement et de recherche français ou étrangers, des laboratoires publics ou privés. 


\title{
Reactive preferential structures and nonmonotonic consequence
}

\author{
Dov M Gabbay * \\ King's College, London ${ }^{\dagger}$ \\ Karl Schlechta $\ddagger$ \\ Laboratoire d'Informatique Fondamentale de Marseille $\S$
}

August 22, 2008

\begin{abstract}
We introduce Information Bearing Relation Systems (IBRS) as an abstraction of many logical systems. We then define a general semantics for IBRS, and show that a special case of IBRS generalizes in a very natural way preferential semantics and solves open representation problems for weak logical systems. This is possible, as we can "break" the strong coherence properties of preferential structures by higher arrows, i.e. arrows, which do not go to points, but to arrows themselves.
\end{abstract}

\section{Contents}

\begin{tabular}{|l|l}
\hline 1 & Introduction
\end{tabular} 2

1.1 Overview . . . . . . . . . . . . . . . . . . . . . . . . 2

1.2 Introduction to IBRS $\ldots \ldots \ldots \ldots \ldots \ldots \ldots \ldots$

1.3 Purpose of this paper $\ldots \ldots \ldots \ldots \ldots \ldots \ldots$

\begin{tabular}{|lll}
2 & A semantics for IBRS & 9 \\
\hline
\end{tabular}

$2.1 \quad$ Introduction $\ldots \ldots \ldots \ldots \ldots \ldots \ldots \ldots$

$2.2 \quad$ Formal definition $\ldots \ldots \ldots \ldots \ldots \ldots \ldots$

$2.3 \quad$ A circuit semantics for simple IBRS without labels . . . . . . . . . . . . . . . . . . . . . 10

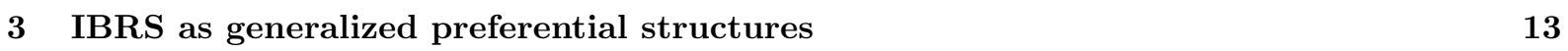

$3.1 \quad$ Introduction . . . . . . . . . . . . . . . . . . . . . . . . . 13

3.2 Generalized preferential structures $\ldots \ldots \ldots \ldots$. . . . . . . . . . . . . . . . 23

3.2.1 Introduction . . . . . . . . . . . . . . . . . . . . . 23

*Dov.Gabbay@kcl.ac.uk, www.dcs.kcl.ac.uk/staff/dg

${ }^{\dagger}$ Department of Computer Science, King's College London, Strand, London WC2R 2LS, UK

${ }^{\ddagger}$ ks@cmi.univ-mrs.fr, karl.schlechta@web.de, http://www.cmi.univ-mrs.fr / ks

$\S$ UMR 6166, CNRS and Université de Provence, Address: CMI, 39, rue Joliot-Curie, F-13453 Marseille Cedex 13, France 
3.2.2 Results on not necessarily smooth structures _ . . . . . . . . . . . . . . . . . . 29

3.2.3 Results on total smoothness . . . . . . . . . . . . . . . . . . . . . 34

3.2 .4 Results on essential smoothness . . . . . . . . . . . . . . . . . . . . . 37

3.2 .5 Translation to logics . . . . . . . . . . . . . . . . . . . . . 42

References 442

\section{Introduction}

\subsection{Overview}

Our aim is twofold here. We want to

(1) introduce IBRS, an abstraction of many semantical structures in nonclassical logics

(2) use IBRS as a generalization of preferential structures to give weaker than preferential logics a semantics.

Many semantical structures for nonclassical logics use sets of models, called possible worlds, together with a relation, e.g. accessibility in the case of Kripke models for modal logic, distance in the case of Stalnaker-Lewis semantics for counterfactual conditionals, or comparison of "normality" in the case of preferential structures. IBRS allow not only relations between objects, i.e. possible worlds, but also "higher" relations, where e.g. an object can be in relation with a pair of objects (which are in a "lower" relation), etc.

It is sometimes natural to see the (basic) relations as attacks: if $a \prec b$, then a is considered more "normal" (or less "abnormal") than $b$, or, we may say that a attacks b's normality, and we may write this as an arrow from a to $b$. When we have now an attack from $c$ against this arrow, i.e. the relation $a \prec b$, it is natural to see now the original attack as destroyed, i.e. a does not attack $b$ any more. So $a \prec b$ is still there, but without any effect. This is possible in an IBRS, see Diagram 1.1. 


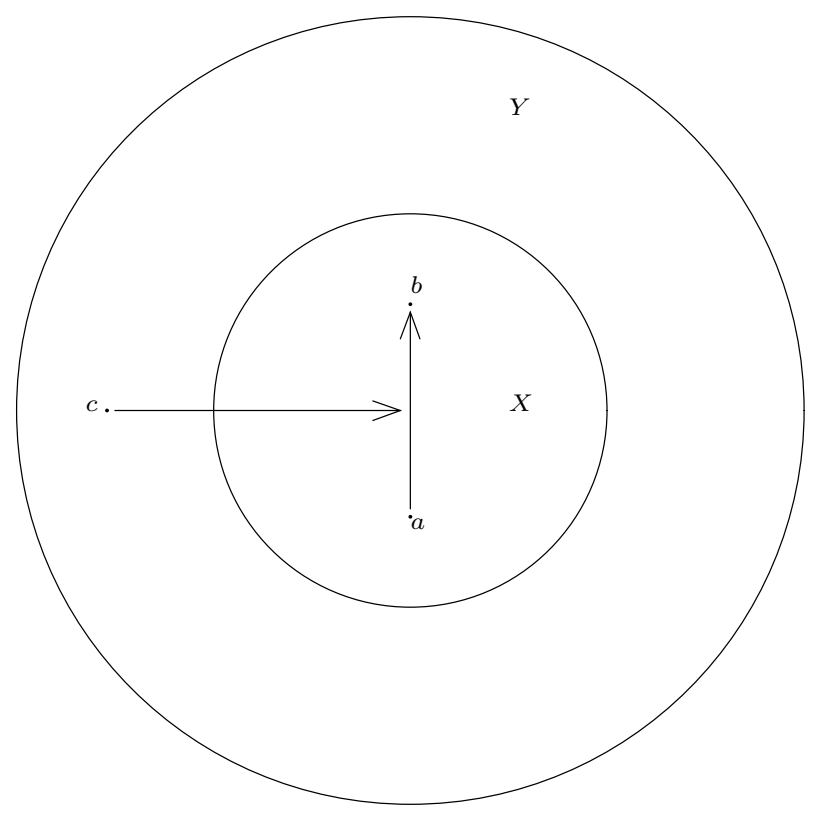

Diagram 1.1

Attacking an arrow

The decisive property of preferential structures is the trivial fact that if $a, b \in X \subseteq Y$, and $a \prec b$ "in $X^{\prime \prime}$,

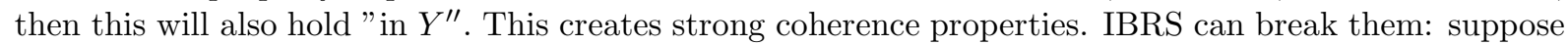
$c \in Y-\mathrm{X}$, and $c$ attacks $a \prec b$, then $a \prec b$ is still there in $Y$, but not effective any more. Thus, IBRS, or generalized preferential structures, as we will call this variant of IBRS, can give a semantics to weaker than preferential logics.

Preferential structures themselves were introduced as abstractions of Circumscription independently in [Sho87b] and BS85]. A precise definition of these structures is given below in Definition 3.5.

In an abstract consideration of desirable properties a logic might have, Gab85 examined rules a nonmonotonic consequence relation $\sim$ should satisfy:

(1) $\Delta, \alpha \sim \alpha$, 
(2) $\Delta \sim \alpha \Rightarrow(\Delta \sim \beta \Leftrightarrow \Delta, \alpha \sim \beta)$.

Both, the semantic and the syntactic, approaches were connected in KLM90, where a representation theorem was proved, showing that the (stronger than Gabbay's) system $P$ corresponds to "smooth" preferential structures. System $P$ consists of

(1) $\phi \sim \psi, \phi \sim \psi^{\prime} \Rightarrow \phi \sim \psi \wedge \psi^{\prime}$,

(2) $\phi \sim \psi, \phi^{\prime} \sim \psi \Rightarrow \phi \vee \phi^{\prime} \sim \psi$,

(3) $\vdash \phi \leftrightarrow \phi^{\prime} \Rightarrow\left(\phi \mid \sim \Leftrightarrow \Leftrightarrow \phi^{\prime} \sim \psi\right)$,

(4) $\phi \vdash \psi, \vdash \psi \rightarrow \psi^{\prime} \Rightarrow \phi \sim \psi^{\prime}$,

(5) $\vdash \phi \rightarrow \phi^{\prime} \Rightarrow \phi \sim \phi^{\prime}$,

(6) $\phi \sim \psi \Rightarrow\left(\phi \sim \psi^{\prime} \Leftrightarrow \phi \wedge \psi \sim \psi^{\prime}\right)$.

where $\vdash$ is classical provability.

Details can be found in Definition 3.4 .

To the authors' knowledge, a precise semantics for Gabbay's system was still lacking. We will give it here, applying the idea of IBRS, which allow to "break" the relations of preferential structures, and thus their strong coherence conditions, as was already illustrated above.

We will also see that the usual definition of "smoothness" - for every arrow from a to $b$, there has to be an arrow from $a^{\prime}$ to $b$, where $a^{\prime}$ is a minimal element - is stronger than needed, and a weaker variant, "essential smoothness" (see Definition 3.14) is sufficient to generate the desired property of cumulativity on the logical side.

\subsection{Introduction to IBRS}

The human agent in his daily activity has to deal with many situations involving change. Chief among them are the following

(1) Common sense reasoning from available data. This involves predication of what unavailable data is supposed to be (nonmonotonic deduction) but it is a defeasible prediction, geared towards immediate change. This is formally known as nonmonotonic reasoning and is studied by the nonmonotonic community.

(2) Belief revision, studied by a very large community. The agent is unhappy with the totality of his beliefs which he finds internally unacceptable (usually logically inconsistent but not necessarily so) and needs to change/revise it.

(3) Receiving and updating his data, studied by the update community.

(4) Making morally correct decisions, studied by the deontic logic community.

(5) Dealing with hypothetical and counterfactual situations. This is studied by a large community of philosophers and AI researchers.

(6) Considering temporal future possibilities, this is covered by modal and temporal logic. 
(7) Dealing with properties that persist through time in the near future and with reasoning that is constructive. This is covered by intuitionistic logic.

All the above types of reasoning exist in the human mind and are used continuously and coherently every hour of the day. The formal modelling of these types is done by diverse communities which are largely distinct with no significant communication or cooperation. The formal models they use are very similar and arise from a more general theory, what we might call:

"Reasoning with information bearing binary relations".

\section{Definition 1.1}

(1) An information bearing binary relation frame IBR, has the form $(S, \Re)$, where $S$ is a non-empty set and $\Re$ is a subset of $S$, where $S$ is defined by induction as follows:

$$
\begin{aligned}
& \text { (1.1) } S_{0}=S \\
& \text { (1.2) } S_{n+1}=S_{n} \cup\left(S_{n} \times S_{n}\right) . \\
& \text { (1.3) } S=\bigcup\left\{S_{n}: n \in \omega\right\}
\end{aligned}
$$

We call elements from $S$ points or nodes, and elements from $\Re$ arrows. Given $(S, \Re)$, we also set $\boldsymbol{P}((S, \Re)):=S$, and $\boldsymbol{A}((S, \Re)):=\Re$.

If $\alpha$ is an arrow, the origin and destination of $\alpha$ are defined as usual, and we write $\alpha: x \rightarrow y$ when $x$ is the origin, and $y$ the destination of the arrow $\alpha$. We also write $o(\alpha)$ and $d(\alpha)$ for the origin and destination of $\alpha$.

(2) Let $Q$ be a set of atoms, and $\boldsymbol{L}$ be a set of labels (usually $\{0,1\}$ or $[0,1]$ ). An information assignment $h$ on $(S, \Re)$ is a function $h: Q \times \Re \rightarrow \boldsymbol{L}$.

(3) An information bearing system IBRS, has the form $(S, \Re, h, Q, \boldsymbol{L})$, where $S, \Re, h, Q, \boldsymbol{L}$ are as above.

See Diagram 1.2 for an illustration. 


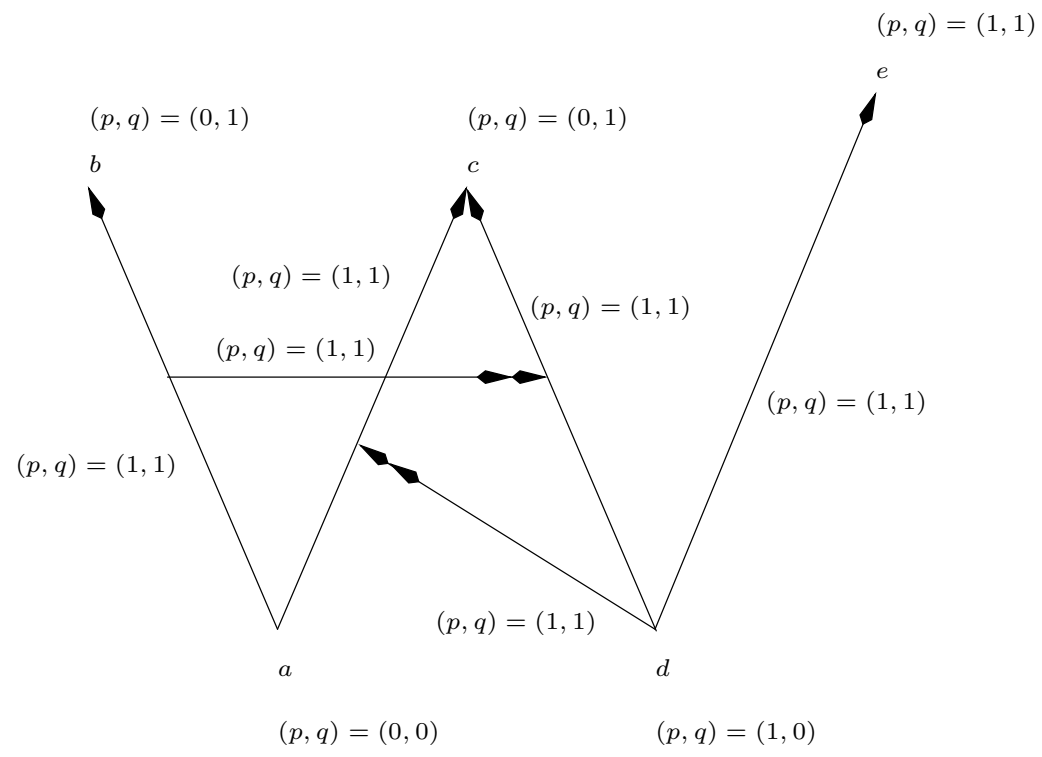

A simple example of an information bearing system.

\section{Diagram 1.2}

We have here:

$$
\begin{aligned}
& S=\{a, b, c, d, e\} . \\
& \Re=S \cup\{(a, b),(a, c),(d, c),(d, e)\} \cup\{((a, b),(d, c)),(d,(a, c))\} . \\
& Q=\{p, q\}
\end{aligned}
$$

The values of $h$ for $p$ and $q$ are as indicated in the figure. For example $h(p,(d,(a, c)))=1$.

\section{Comment 1.1}

The elements in Figure Diagram 1.2 can be interpreted in many ways, depending on the area of application.

(1) The points in $S$ can be interpreted as possible worlds, or as nodes in an argumentation network or nodes in a neural net or states, etc.

(2) The direct arrows from nodes to nodes can be interpreted as accessibility relation, attack or support arrows in an argumentation networks, connection in a neural nets, a preferential ordering in a nonmonotonic model, etc.

(3) The labels on the nodes and arrows can be interpreted as fuzzy values in the accessibility relation or weights in the neural net or strength of arguments and their attack in argumentation nets, or distances in a counterfactual model, etc.

(4) The double arrows can be interpreted as feedback loops to nodes or to connections. 
Thus, IBRS can be used as a source of information for various logics based on the atoms in $Q$. We now illustrate by listing several such logics.

\section{Modal Logic}

One can consider the figure as giving rise to two modal logic models. One with actual world a and one with $d$, these being the two minimal points of the relation. Consider a language with $\square q$. how do we evaluate $a \models \square q$ ?

The modal logic will have to give an algorithm for calculating the values.

Say we choose algorithm $\mathcal{A}_{1}$ for $a \models \square q$, namely:

$\left[\mathcal{A}_{1}(a, \square q)=1\right]$ iff for all $x \in S$ such that $a=x$ or $(a, x) \in \Re$ we have $h(q, x)=1$.

According to $\mathcal{A}_{1}$ we get that $\square q$ is false at a. $\mathcal{A}_{1}$ gives rise to a $T$-modal logic. Note that the reflexivity is not anchored at the relation $\Re$ of the network but in the algorithm $\mathcal{A}_{1}$ in the way we evaluate. We say $(S, \Re, \ldots) \models \square q$ iff $\square q$ holds in all minimal points of $(S, \Re)$.

For orderings without minimal points we may choose a subset of distinguished points.

\section{Nonmonotonic Deduction}

We can ask whether $p \sim q$ according to algorithm $\mathcal{A}_{2}$ defined below. $\mathcal{A}_{2}$ says that $p \sim q$ holds iff $q$ holds in all minimal models of $p$. Let us check the value of $\mathcal{A}_{2}$ in this case:

Let $S_{p}=\{s \in S \mid h(p, s)=1\}$. Thus $S_{p}=\{d, e\}$.

The minimal points of $S_{p}$ are $\{d\}$. Since $h(q, d)=0$, we have that $p \not \chi q$.

Note that in the cases of modal logic and nonmonotonic logic we ignored the arrows $(d,(a, c))$ (i.e. the double arrow from $d$ to the arc $(a, c)$ ) and the $h$ values to arcs. These values do not play a part in the traditional modal or nonmonotonic logic. They do play a part in other logics. The attentive reader may already suspect that we have her an opportunity for generalisation of say nonmonotonic logic, by giving a role to arc annotations.

\section{Argumentation Nets}

Here the nodes of $S$ are interpreted as arguments. The atoms $\{p, q\}$ can be interpreted as types of arguments and the arrows e.g. $(a, b) \in \Re$ as indicating that the argument a is attacking the argument $b$.

So, for example, let

$\mathrm{a}=$ we must win votes.

$b=$ death sentence for murderers.

$c=$ We must allow abortion for teenagers

$d=$ Bible forbids taking of life.

$q=$ the argument is a social argument

$p=$ the argument is a religious argument.

$(d,(a, c))=$ there should be no connection between winning votes and abortion.

$((a, b),(d, c))=$ If we attack the death sentence in order to win votes then we must stress

(attack) that there should be no connection between religion (Bible) and social issues. 
Thus we have according to this model that supporting abortion can lose votes. The argument for abortion is a social one and the argument from the Bible against it is a religious one.

We can extract information from this IBRS using two algorithms. The modal logic one can check whether for example every social argument is attacked by a religious argument. The answer is no, since the social $\operatorname{argument} b$ is attacked only by a which is not a religious argument.

We can also use algorithm $\mathcal{A}_{3}$ (following Dung) to extract the winning arguments of this system. The arguments a and $d$ are winning since they are not attacked. $d$ attacks the connection between a and $c$ (i.e. stops a attacking $c$ ).

The attack of a on $b$ is successful and so $b$ is out. However the $\operatorname{arc}(a, b)$ attacks the $\operatorname{arc}(d, c)$. So $c$ is not attacked at all as both arcs leading into it are successfully eliminated. So $c$ is in. $e$ is out because it is attacked by $d$.

So the winning arguments are $\{a, c, d\}$

In this model we ignore the annotations on arcs. To be consistent in our mathematics we need to say that $h$ is a partial function on $\Re$. The best way is to give more specific definition on IBRS to make it suitable for each logic.

\section{Counterfactuals}

The traditional semantics for counterfactuals involves closeness of worlds. The clauses $y \models p \hookrightarrow q$, where $\hookrightarrow$ is a counterfactual implication is that $q$ holds in all worlds $y^{\prime}$ "near enough" to $y$ in which $p$ holds. So if we interpret the annotation on arcs as distances then we can define "near" as distance $\leq 2$, we get: $a \models p \hookrightarrow q$ iff in all worlds of $p$-distance $\leq 2$ if $p$ holds so does $q$. Note that the distance depends on $p$. In this case we get that $a \models p \hookrightarrow q$ holds. The distance function can also use the arrows from arcs to arcs, etc. There are many opportunities for generalisation in our IBRS set up.

\section{Intuitionistic Persistence}

We can get an intuitionistic Kripke model out of this IBRS by letting, for $t, s \in S, t \rho_{0} s$ iff $t=s$ or $[t R s \wedge \forall q \in Q(h(q, t) \leq h(q, s))]$. We get that

$\left[r_{0}=\{(y, y) \mid y \in S\} \cup\{(a, b),(a, c),(d, e)\}.\right]$

Let $\rho$ be the transitive closure of $\rho_{0}$. Algorithm $\mathcal{A}_{4}$ evaluates $p \Rightarrow q$ in this model, where $\Rightarrow$ is intuitionistic implication.

$\mathcal{A}_{4}: p \Rightarrow q$ holds at the IBRS iff $p \Rightarrow q$ holds intuitionistically at every $\rho$-minimal point $o f(S, \rho)$.

\subsection{Purpose of this paper}

In this paper, we will not cover all these applications of the above abstract definition of IBRS, but will

(1) give an abstract and also a very concrete semantics to IBRS

(2) show that a special case of IBRS generalizes in a very natural way preferential semantics and solves open representation problems for weak logical systems. This is possible, as we can "break" the strong coherence properties of preferential structures by higher arrows, i.e. arrows, which do not go to points, but to arrows themselves. 


\section{$2 \quad$ A semantics for IBRS}

\section{$2.1 \quad$ Introduction}

(1) Nodes and arrows

As we may have counterarguments not only against nodes, but also against arrows, they must be treated basically the same way, i.e. in some way there has to be a positive, but also a negative influence on both. So arrows cannot just be concatenation between the contents of nodes, or so.

We will differentiate between nodes and arrows by labelling arrows in addition with a time delay. We see nodes as situations, where the output is computed instantenously from the input, whereas arrows describe some "force" or "mechanism" which may need some time to "compute" the result from the input.

Consequently, if $\alpha$ is an arrow, and $\beta$ an arrow pointing to $\alpha$, then it should point to the input of $\alpha$, i.e. before the time lapse. Conversely, any arrow originating in $\alpha$ should originate after the time lapse.

Apart this distinction, we will treat nodes and arrows the same way, so the following discussion will apply to both - which we call just "objects".

(2) Defeasibility

The general idea is to code each object, say $X$, by $I(X): U(X) \rightarrow C(X):$ If $I(X)$ holds then, unless $U(X)$ holds, consequence $C(X)$ will hold. (We adopted Reiter's notation for defaults, as IBRS have common points with the former.)

The situation is slightly more complicated, as there can be several counterarguments, so $U(X)$ really is an "or". Likewise, there can be several supporting arguments, so $I(X)$ also is an "or".

A counterargument must not always be an argument against a specific supporting argument, but it can be. Thus, we should admit both possibilties. As we can use arrows to arrows, the second case is easy to treat (as is the dual, a supporting argument can be against a specific counterargument). How do we treat the case of unspecific pro- and counterarguments? Probably the easiest way is to adopt Dung's idea: an object is in, if it has at least one support, and no counterargument - see [Dun95]. Of course, other possibilities may be adopted, counting, use of labels, etc., but we just consider the simple case here.

(3) Labels

In the general case, objects stand for some kind of defeasible transmission. We may in some cases see labels as restricting this transmission to certain values. For instance, if the label is $p=1$ and $q=0$, then the $p$-part may be transmitted and the $q$-part not.

Thus, a transmission with a label can sometimes be considered as a family of transmissions, which ones are active is indicated by the label.

\section{Example 2.1}

In fuzzy Kripke models, labels are elements of $[0,1] \cdot p=0.5$ as label for a node $m^{\prime}$ which stands for a fuzzy model means that the value of $p$ is 0.5. $p=0.5$ as label for an arrow from $m$ to $m^{\prime}$ means that $p$ is transmitted with value 0.5 . Thus, when we look from $m$ to $m^{\prime}$, we see $p$ with value $0.5 * 0.5=0.25$. So, we have $\diamond p$ with value 0.25 at $m$ - if, e.g., $m, m^{\prime}$ are the only models.

(4) Putting things together

If an arrow leaves an object, the object's output will be connected to the (only) positive input of the arrow. (An arrow has no negative inputs from objects it leaves.) If a positive arrow enters an object, it is connected to one of the positive inputs of the object, analogously for negative arrows and inputs.

When labels are present, they are transmitted through some operation. 


\section{$2.2 \quad$ Formal definition}

\section{Definition 2.1}

In the most general case, objects of IBRS have the form: $\left(<I_{1}, L_{1}>, \ldots,<I_{n}, L_{n}>\right):\left(<U_{1}, L_{1}^{\prime}>\right.$ $, \ldots,<U_{n}, L_{n}^{\prime}>$ ), where the $L_{i}, L_{i}^{\prime}$ are labels and the $I_{i}, U_{i}$ might be just truth values, but can also be more complicated, a (possibly infinite) sequence of some values. Connected objects have, of course, to have corresponding such sequences. In addition, the object $X$ has a criterion for each input, whether it is valid or not (in the simple case, this will just be the truth value "true"). If there is at least one positive valid input $I_{i}$, and no valid negative input $U_{i}$, then the output $C(X)$ and its label are calculated on the basis of the valid inputs and their labels. If the object is an arrow, this will take some time, $t$, otherwise, this is instantaneous.

\section{Evaluating a diagram}

An evaluation is relative to a fixed input, i.e. some objects will be given certain values, and the diagram is left to calculate the others. It may well be that it oscillates, i.e. shows a cyclic behaviour. This may be true for a subset of the diagram, or the whole diagram. If it is restricted to an unimportant part, we might neglect this. Whether it oscillates or not can also depend on the time delays of the arrows (see Example 2.2).

We therefore define for a diagram $\Delta$

$\alpha \vdash{ }_{\Delta} \beta$ iff

(a) $\alpha$ is a (perhaps partial) input - where the other values are set "not valid"

(b) $\beta$ is a (perhaps partial) output

(c) after some time, $\beta$ is stable, i.e. all still possible oscillations do not affect $\beta$

(d) the other possible input values do not matter, i.e. whatever the input, the result is the same.

In the cases examined here more closely, all input values will be defined.

\subsection{A circuit semantics for simple IBRS without labels}

It is standard to implement the usual logical connectives by electronic circuits. These components are called gates. Circuits with feedback sometimes show undesirable behaviour when the initial conditions are not specified. (When we switch a circuit on, the outputs of the individual gates can have arbitrary values.) The technical realization of these initial values shows the way to treat defaults. The initial values are set via resistors (in the order of $1 \mathrm{k} \Omega$ ) between the point in the circuit we want to intialize and the desired tension (say 0 Volt for false, 5 Volt for true). They are called pull-down or pull-up resistors (for default 0 or 5 Volt). When a "real" result comes in, it will override the tension applied via the resistor.

Closer inspection reveals that we have here a 3 level default situation: The initial value will be the weakest, which can be overridden by any "real" signal, but a positive argument can be overridden by a negative one. Thus, the biggest resistor will be for the initialization, the smaller one for the supporting arguments, and the negative arguments have full power.

Technical details will be left to the experts.

We give now an example which shows that the delays of the arrows can matter. In one situation, a stable state is reached, in another, the circuit begins to oscillate. 


\section{Example 2.2}

(In engineering terms, this is a variant of a JK flip-flop with $R * S=0$, a circuit with feedback.)

We have 8 measuring points.

In 1, In 2 are the overall input, Out1, Out2 the overall output, $A 1, A 2, A 3, A 4$ are auxiliary internal points. All points can be true or false.

The logical structure is as follows:

$\mathrm{A} 1=\operatorname{In} 1 \wedge$ Out $1, \mathrm{~A} 2=\operatorname{In} 2 \wedge$ Out 2 ,

$\mathrm{A} 3=A 1 \vee$ Out $2, \mathrm{~A} 4=A 2 \vee$ Out 1 ,

Out1 $=\neg A 3$, Out2 $=\neg A 4$.

Thus, the circuit is symmetrical, with In1 corresponding to In2, A1 to A2, A3 to A4, Out1 to Out2.

The input is held constant. See Diagram 2.1.

Diagram 2.1 


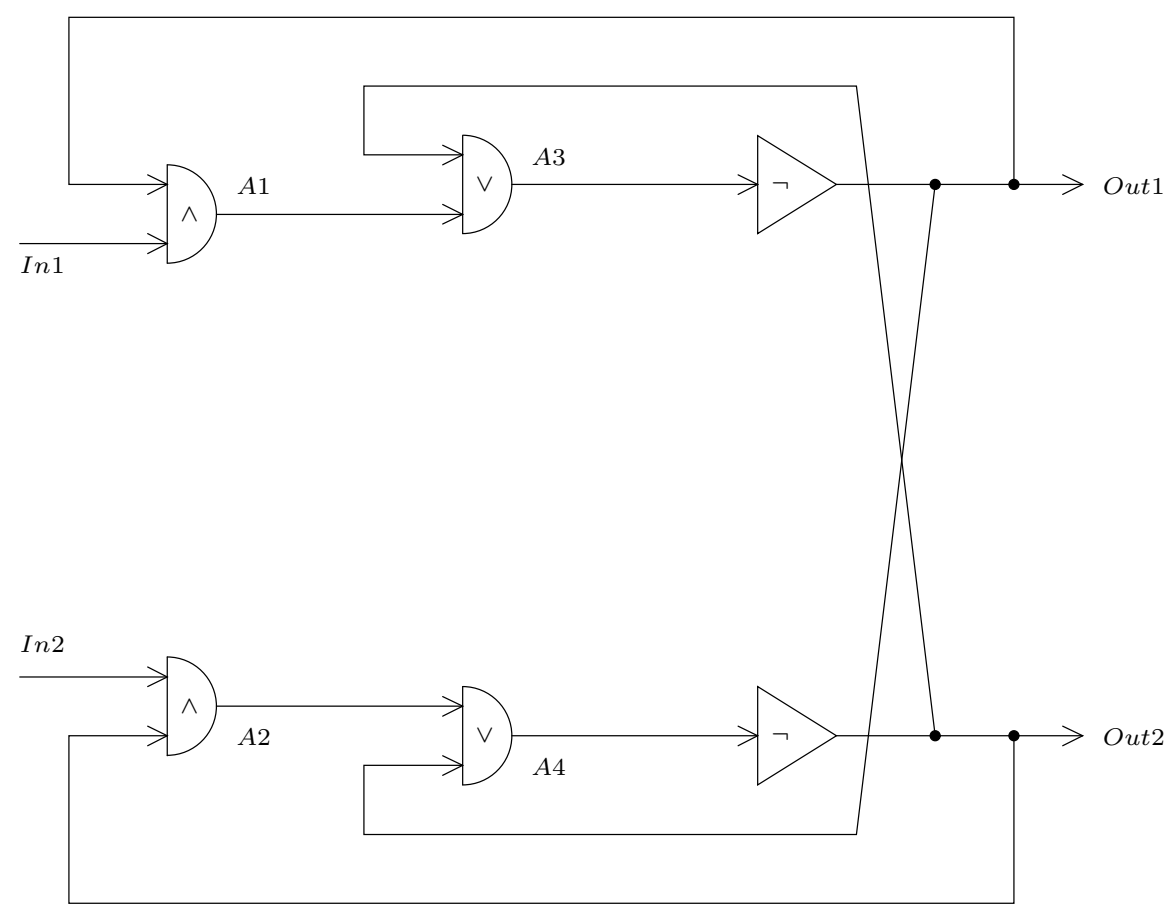

Gate Semantics

We suppose that the output of the individual gates is present $n$ time slices after the input was present. $n$ will in the first circuit be equal to 1 for all gates, in the second circuit equal to 1 for all but the AND gates, which will take 2 time slices. Thus, in both cases, e.g. Out1 at time $t$ will be the negation of A3 at time $t-1$. In the first case, A1 at time $t$ will be the conjunction of In1 and Out1 at time $t-1$, and in the second case the conjunction of In1 and Out1 at time $t-2$.

We initialize In1 as true, all others as false. (The initial value of A3 and A4 does not matter, the behaviour 
is essentially the same for all such values.)

The first circuit will oscillate with a period of 4 , the second circuit will go to a stable state.

We have the following transition tables (time slice shown at left):

Circuit 1, delay $=1$ everywhere:

$\begin{array}{cccccccccc} & \text { In1 } & \text { In2 } & \text { A1 } & \text { A2 } & \text { A3 } & \text { A4 } & \text { Out1 } & \text { Out2 } & \\ \text { 1: } & \mathrm{T} & \mathrm{F} & \mathrm{F} & \mathrm{F} & \mathrm{F} & \mathrm{F} & \mathrm{F} & \mathrm{F} & \\ 2: & \mathrm{T} & \mathrm{F} & \mathrm{F} & \mathrm{F} & \mathrm{F} & \mathrm{F} & \mathrm{T} & \mathrm{T} & \\ \text { 3: } & \mathrm{T} & \mathrm{F} & \mathrm{T} & \mathrm{F} & \mathrm{T} & \mathrm{T} & \mathrm{T} & \mathrm{T} & \\ \text { 4: } & \mathrm{T} & \mathrm{F} & \mathrm{T} & \mathrm{F} & \mathrm{T} & \mathrm{T} & \mathrm{F} & \mathrm{F} & \\ 5: & \mathrm{T} & \mathrm{F} & \mathrm{F} & \mathrm{F} & \mathrm{T} & \mathrm{F} & \mathrm{F} & \mathrm{F} & \text { oscillation starts } \\ 6: & \mathrm{T} & \mathrm{F} & \mathrm{F} & \mathrm{F} & \mathrm{F} & \mathrm{F} & \mathrm{F} & \mathrm{T} & \\ 7: & \mathrm{T} & \mathrm{F} & \mathrm{F} & \mathrm{F} & \mathrm{T} & \mathrm{F} & \mathrm{T} & \mathrm{T} & \\ \text { 8: } & \mathrm{T} & \mathrm{F} & \mathrm{T} & \mathrm{F} & \mathrm{T} & \mathrm{T} & \mathrm{F} & \mathrm{T} & \\ 9: & \mathrm{T} & \mathrm{F} & \mathrm{F} & \mathrm{F} & \mathrm{T} & \mathrm{F} & \mathrm{F} & \mathrm{F} & \text { back to start of oscillation }\end{array}$

Circuit 2, delay $=1$ everywhere, except for AND with delay $=2$ :

(Thus, A1 and A2 are held at their intial value up to time 2, then they are calculated using the values of time $t-2$.)

$\begin{array}{cccccccccc} & \text { In1 } & \text { In2 } & \text { A1 } & \text { A2 } & \text { A3 } & \text { A4 } & \text { Out1 } & \text { Out2 } \\ \text { 1: } & \text { T } & \text { F } & \text { F } & \text { F } & \text { F } & \text { F } & \text { F } & \text { F } \\ \text { 2: } & \text { T } & \text { F } & \text { F } & \text { F } & \text { F } & \text { F } & \text { T } & \text { T } \\ \text { 3: } & \mathrm{T} & \mathrm{F} & \mathrm{F} & \mathrm{F} & \mathrm{T} & \mathrm{T} & \mathrm{T} & \mathrm{T} & \\ \text { 4: } & \mathrm{T} & \mathrm{F} & \mathrm{T} & \mathrm{F} & \mathrm{T} & \mathrm{T} & \mathrm{F} & \mathrm{F} & \\ 5: & \mathrm{T} & \mathrm{F} & \mathrm{T} & \mathrm{F} & \mathrm{T} & \mathrm{F} & \mathrm{F} & \mathrm{F} & \\ 6: & \mathrm{T} & \mathrm{F} & \mathrm{F} & \mathrm{F} & \mathrm{T} & \mathrm{F} & \mathrm{F} & \mathrm{T} & \text { stable state reached } \\ \text { 7: } & \mathrm{T} & \mathrm{F} & \mathrm{F} & \mathrm{F} & \mathrm{T} & \mathrm{F} & \mathrm{F} & \mathrm{T} & \end{array}$

Note that state 6 of circuit 2 is also stable in circuit 1, but it is never reached in that circuit.

\section{IBRS as generalized preferential structures}

\subsection{Introduction}

\section{Definition 3.1}

We use $\mathcal{P}$ to denote the power set operator, $\Pi\left\{X_{i}: i \in I\right\}:=\left\{g: g: I \rightarrow \bigcup\left\{X_{i}: i \in I\right\}, \forall i \in\right.$ $\left.I . g(i) \in X_{i}\right\}$ is the general cartesian product, $\operatorname{card}(X)$ shall denote the cardinality of $X$, and $V$ the set-theoretic universe we work in - the class of all sets. Given a set of pairs $\mathcal{X}$, and a set $X$, we denote by $\mathcal{X}\lceil X:=\{\langle x, i>\in \mathcal{X}: x \in X\}$. When the context is clear, we will sometime simply write $X$ for $\mathcal{X}\lceil X$.

$A \subseteq B$ will denote that $A$ is a subset of $B$ or equal to $B$, and $A \subset B$ that $A$ is a proper subset of $B$, likewise for $A \supseteq B$ and $A \supset B$.

Given some fixed set $U$ we work in, and $X \subseteq U$, then $C(X):=U-X$.

If $\mathcal{Y} \subseteq \mathcal{P}(X)$ for some $X$, we say that $\mathcal{Y}$ satisfies 
$(\cap)$ iff it is closed under finite intersections,

(П) iff it is closed under arbitrary intersections,

$(\cup)$ iff it is closed under finite unions,

(U) iff it is closed under arbitrary unions,

$(\boldsymbol{C})$ iff it is closed under complementation.

We will sometimes write $A=B \| C$ for: $A=B$, or $A=C$, or $A=B \cup C$.

We make ample and tacit use of the Axiom of Choice.

\section{Definition 3.2}

$\prec^{*}$ will denote the transitive closure of the relation $\prec$. If a relation $<, \prec$, or similar is given, $a \perp b$ will express that a and $b$ are $<-($ or $\prec-)$ incomparable - context will tell. Given any relation $<$, $\leq$ will stand for $<$ or $=$, conversely, given $\leq$, $<$ will stand for $\leq$, but not $=$, similarly for $\prec$ etc.

\section{Definition 3.3}

We work here in a classical propositional language $\mathcal{L}$, a theory $T$ will be an arbitrary set of formulas. Formulas will often be named $\phi, \psi$, etc., theories $T, S$, etc.

$v(\mathcal{L})$ will be the set of propositional variables of $\mathcal{L}$.

$M_{\mathcal{L}}$ will be the set of (classical) models of $\mathcal{L}, M(T)$ or $M_{T}$ is the set of models of $T$, likewise $M(\phi)$ for a formula $\phi$.

$\boldsymbol{D}_{\mathcal{L}}:=\{M(T): T$ a theory in $\mathcal{L}\}$, the set of definable model sets.

Note that, in classical propositional logic, $\emptyset, M_{\mathcal{L}} \in \boldsymbol{D}_{\mathcal{L}}, \boldsymbol{D}_{\mathcal{L}}$ contains singletons, is closed under arbitrary intersections and finite unions.

An operation $f: \mathcal{Y} \rightarrow \mathcal{P}\left(M_{\mathcal{L}}\right)$ for $\mathcal{Y} \subseteq \mathcal{P}\left(M_{\mathcal{L}}\right)$ is called definability preserving, $(d p)$ or $(\mu d p)$ in short, iff for all $X \in \boldsymbol{D}_{\mathcal{L}} \cap \mathcal{Y} f(X) \in \boldsymbol{D}_{\mathcal{L}}$.

We will also use $(\mu d p)$ for binary functions $f: \mathcal{Y} \times \mathcal{Y} \rightarrow \mathcal{P}\left(M_{\mathcal{L}}\right)$ - as needed for theory revision - with the obvious meaning.

$\vdash$ will be classical derivability, and

$\bar{T}:=\{\phi: T \vdash \phi\}$, the closure of $T$ under $\vdash$.

$\operatorname{Con}($.$) will stand for classical consistency, so \operatorname{Con}(\phi)$ will mean that $\phi$ is clasical consistent, likewise for $\operatorname{Con}(T)$. $\operatorname{Con}\left(T, T^{\prime}\right)$ will stand for $\operatorname{Con}\left(T \cup T^{\prime}\right)$, etc.

Given a consequence relation $\sim$, we define

$\overline{\bar{T}}:=\{\phi: T \sim \phi\}$.

(There is no fear of confusion with $\bar{T}$, as it just is not useful to close twice under classical logic.)

$T \vee T^{\prime}:=\left\{\phi \vee \phi^{\prime}: \phi \in T, \phi^{\prime} \in T^{\prime}\right\}$.

If $X \subseteq M_{\mathcal{L}}$, then $T h(X):=\{\phi: X \models \phi\}$, likewise for $T h(m), m \in M_{\mathcal{L}}$.

We recollect and note:

\section{Fact 3.1}

Let $\mathcal{L}$ be a fixed propositional language, $\boldsymbol{D}_{\mathcal{L}} \subseteq X, \mu: X \rightarrow \mathcal{P}\left(M_{\mathcal{L}}\right)$, for a $\mathcal{L}$-theory $T \overline{\bar{T}}:=T h\left(\mu\left(M_{T}\right)\right)$, let $T, T^{\prime}$ be arbitrary theories, then: 
(1) $\mu\left(M_{T}\right) \subseteq M_{\overline{\bar{T}}}$,

(2) $M_{T} \cup M_{T^{\prime}}=M_{T \vee T^{\prime}}$ and $M_{T \cup T^{\prime}}=M_{T} \cap M_{T^{\prime}}$,

(3) $\mu\left(M_{T}\right)=\emptyset \leftrightarrow \perp \in \overline{\bar{T}}$.

If $\mu$ is definability preserving or $\mu\left(M_{T}\right)$ is finite, then the following also hold:

(4) $\mu\left(M_{T}\right)=M_{\overline{\bar{T}}}$,

(5) $T^{\prime} \vdash \overline{\bar{T}} \leftrightarrow M_{T^{\prime}} \subseteq \mu\left(M_{T}\right)$,

(6) $\mu\left(M_{T}\right)=M_{T^{\prime}} \leftrightarrow \overline{T^{\prime}}=\overline{\bar{T}}$.

\section{Definition 3.4}

We introduce here formally a list of properties of set functions on the algebraic side, and their corresponding logical rules on the other side.

Recall that $\bar{T}:=\{\phi: T \vdash \phi\}, \overline{\bar{T}}:=\{\phi: T \sim \phi\}$, where $\vdash$ is classical consequence, and $\sim$ any other consequence.

We show, wherever adequate, in parallel the formula version in the left column, the theory version in the middle column, and the semantical or algebraic counterpart in the right column. The algebraic counterpart gives conditions for a function $f: \mathcal{Y} \rightarrow \mathcal{P}(U)$, where $U$ is some set, and $\mathcal{Y} \subseteq \mathcal{P}(U)$.

When the formula version is not commonly used, we omit it, as we normally work only with the theory version.

Intuitively, $A$ and $B$ in the right hand side column stand for $M(\phi)$ for some formula $\phi$, whereas $X, Y$ stand for $M(T)$ for some theory $T$. 


\begin{tabular}{|c|c|c|}
\hline \multicolumn{3}{|c|}{ Basics } \\
\hline $\begin{array}{c}(A N D) \\
\phi \sim \psi, \phi \sim \psi^{\prime} \Rightarrow \\
\phi \sim \psi \wedge \psi^{\prime}\end{array}$ & $\begin{array}{c}(A N D) \\
T \sim \psi, T \sim \psi^{\prime} \Rightarrow \\
T \sim \psi \wedge \psi^{\prime}\end{array}$ & $\begin{array}{l}\text { Closure under } \\
\text { finite } \\
\text { intersection } \\
\end{array}$ \\
\hline $\begin{array}{c}(O R) \\
\phi \sim \psi, \phi^{\prime} \sim \psi \Rightarrow \\
\phi \vee \phi^{\prime} \sim \psi\end{array}$ & $\overline{\bar{T} \cap \overline{\overline{T^{\prime}}} \subseteq \overline{\overline{T \vee T^{\prime}}}}$ & $\begin{array}{c}(\mu O R) \\
f(X \cup Y) \subseteq f(X) \cup f(Y)\end{array}$ \\
\hline $\begin{array}{c}(w O R) \\
\phi \sim \psi, \phi^{\prime} \vdash \psi \Rightarrow \\
\phi \vee \phi^{\prime} \sim \psi\end{array}$ & $\overline{\bar{T} \cap \overline{(w O R)}} \overline{\overline{T^{\prime}} \subseteq \overline{\overline{T \vee T^{\prime}}}}$ & $\begin{array}{c}(\mu w O R) \\
f(X \cup Y) \subseteq f(X) \cup Y\end{array}$ \\
\hline $\begin{array}{c}(\operatorname{disjOR)} \\
\phi \vdash \neg \phi^{\prime}, \phi \sim \psi, \\
\phi^{\prime} \sim \psi \Rightarrow \phi \vee \phi^{\prime} \sim \psi \\
\end{array}$ & $\begin{array}{c}(\operatorname{disjOR}) \\
\neg \operatorname{Con}\left(T \cup T^{\prime}\right) \Rightarrow \\
\overline{\bar{T}} \overline{\overline{T^{\prime}}} \subseteq \overline{\overline{T \vee T^{\prime}}} \\
\end{array}$ & $\begin{array}{c}(\mu \text { disjOR }) \\
X \cap Y=\emptyset \Rightarrow \\
f(X \cup Y) \subseteq f(X) \cup f(Y) \\
\end{array}$ \\
\hline $\begin{array}{c}(L L E) \\
\text { Left Logical Equivalence } \\
\vdash \phi \leftrightarrow \phi^{\prime}, \phi \sim \psi \Rightarrow \\
\phi^{\prime}, \sim \psi\end{array}$ & $\begin{array}{c}(L \overline{L E}) \\
\bar{T}=\overline{T^{\prime}} \Rightarrow \overline{\bar{T}}=\overline{\overline{T^{\prime}}}\end{array}$ & trivially true \\
\hline $\begin{array}{c}(R W) \text { Right Weakening } \\
\phi \sim \psi, \vdash \psi \rightarrow \psi^{\prime} \Rightarrow \\
\phi \mid \sim \psi^{\prime}\end{array}$ & $\begin{array}{c}(R W) \\
T \sim \psi, \vdash \psi \rightarrow \psi^{\prime} \Rightarrow \\
T \sim \psi^{\prime}\end{array}$ & upward closure \\
\hline$(C C L)$ Classical Closure & $\begin{array}{c}(C C L) \\
\overline{\bar{T}} \text { is classically } \\
\text { closed }\end{array}$ & trivially true \\
\hline $\begin{array}{c}(S C) \text { Supraclassicality } \\
\phi \vdash \psi \Rightarrow \phi \sim \psi \\
(R E F) \text { Reflexivity } \\
\Delta, \alpha \sim \alpha\end{array}$ & $\begin{array}{l}(S C) \\
\bar{T} \subseteq \overline{\bar{T}}\end{array}$ & $\begin{array}{c}(\mu \subseteq) \\
f(X) \subseteq X\end{array}$ \\
\hline $\begin{array}{c}(C P) \\
\text { Consistency Preservation } \\
\phi \sim \perp \Rightarrow \phi \vdash \perp \\
\end{array}$ & $\begin{array}{c}(C P) \\
T \sim \perp \Rightarrow T \vdash \perp\end{array}$ & $\begin{array}{c}(\mu \emptyset) \\
f(X)=\emptyset \Rightarrow X=\emptyset\end{array}$ \\
\hline & & $\begin{array}{c}(\mu \emptyset \text { fin }) \\
X \neq \emptyset \Rightarrow f(X) \neq \emptyset \\
\text { for finite } X\end{array}$ \\
\hline$\overline{\overline{\phi \wedge \phi^{\prime}}} \subseteq \overline{\overline{\bar{\phi}} \cup\left\{\phi^{\prime}\right\}}$ & $\begin{array}{c}\overline{(P R)} \\
\overline{\overline{T \cup T^{\prime}}} \subseteq \overline{\bar{T} \cup T^{\prime}}\end{array}$ & $\begin{array}{c}(\mu P R) \\
X \subseteq Y \Rightarrow \\
f(Y) \cap X \subseteq f(X)\end{array}$ \\
\hline & & $\begin{array}{c}\left(\mu P R^{\prime}\right) \\
f(X) \cap Y \subseteq f(X \cap Y)\end{array}$ \\
\hline $\begin{array}{c}(C U T) \\
\Delta \sim \alpha ; \Delta, \alpha \sim \beta \Rightarrow \\
\Delta \sim \beta\end{array}$ & $\begin{array}{c}(C U T) \\
T \subseteq \overline{T^{\prime}} \subseteq \overline{\bar{T}} \Rightarrow \\
\overline{\overline{T^{\prime}}} \subseteq \overline{\bar{T}}\end{array} \Rightarrow$ & $\begin{array}{c}(\mu C U T) \\
f(X) \subseteq Y \subseteq X \Rightarrow \\
f(X) \subseteq f(Y)\end{array}$ \\
\hline
\end{tabular}




\begin{tabular}{|c|c|c|}
\hline \multicolumn{3}{|c|}{ Cumulativity } \\
\hline$(C M)$ Cautious Monotony & \multirow{4}{*}{$\begin{array}{c}(C M) \\
T \subseteq \overline{T^{\prime}} \subseteq \overline{\bar{T}} \\
\overline{\bar{T}} \subseteq \overline{\overline{T^{\prime}}}\end{array} \Rightarrow$} & $(\mu C M)$ \\
\hline$\phi \sim \psi, \phi \mid \sim \psi^{\prime} \Rightarrow$ & & $f(X) \subseteq Y \subseteq X \Rightarrow$ \\
\hline$\phi \wedge \psi \mid \sim \psi^{\prime}$ & & $f(Y) \subseteq f(X)$ \\
\hline $\begin{array}{c}\text { or }(\text { ResM) Restricted Monotony } \\
\Delta \sim \sim \alpha, \beta \Rightarrow \Delta, \alpha \sim \beta\end{array}$ & & $\begin{array}{c}(\mu \operatorname{Res} M) \\
f(X) \subseteq A \cap B \Rightarrow f(X \cap A) \subseteq B\end{array}$ \\
\hline$(C U M)$ Cumulativity & $(C U M)$ & $(\mu C U M)$ \\
\hline $\begin{array}{c}\phi \sim \psi \Rightarrow \\
\left(\phi \sim \psi^{\prime} \Leftrightarrow \phi \wedge \psi \sim \psi^{\prime}\right)\end{array}$ & $\begin{array}{c}T \subseteq \overline{T^{\prime}} \subseteq \overline{\bar{T}} \\
\overline{\bar{T}}=\overline{\overline{T^{\prime}}}\end{array} \Rightarrow$ & $\begin{array}{c}f(X) \subseteq Y \subseteq X \Rightarrow \\
f(Y)=f(X)\end{array}$ \\
\hline & $\begin{array}{c}(\subseteq \supseteq) \\
T \subseteq \overline{\overline{T^{\prime}}}, T^{\prime} \subseteq \overline{\bar{T}} \Rightarrow \\
\overline{\overline{T^{\prime}}}=\overline{\bar{T}}\end{array}$ & $\begin{array}{c}(\mu \subseteq \supseteq) \\
f(X) \subseteq Y, f(Y) \subseteq X \Rightarrow \\
f(X)=f(Y)\end{array}$ \\
\hline \multicolumn{3}{|c|}{ Rationality } \\
\hline $\begin{array}{c}\text { RatM) Rational Monotony } \\
\phi \sim \psi, \phi \not \psi \neg \psi^{\prime} \Rightarrow \\
\phi \wedge \psi^{\prime} \sim \psi\end{array}$ & $\begin{array}{c}(\text { Rat M) } \\
\operatorname{Con}\left(T \cup \overline{\overline{T^{\prime}}}\right), T \vdash T^{\prime} \Rightarrow \\
\overline{\bar{T}} \supseteq \overline{\overline{\overline{T^{\prime}}} \cup T}\end{array}$ & $\begin{array}{c}(\mu \text { RatM }) \\
X \subseteq Y, X \cap f(Y) \neq \emptyset \Rightarrow \\
f(X) \subseteq f(Y) \cap X\end{array}$ \\
\hline & $\begin{array}{c}(\overline{R a t M}=) \\
\operatorname{Con}\left(T \cup \overline{\overline{T^{\prime}}}\right), T \vdash T^{\prime} \Rightarrow \\
\overline{\bar{T}}=\overline{\overline{\overline{T^{\prime}}} \cup T}\end{array}$ & $\begin{array}{c}(\mu=) \\
X \subseteq Y, X \cap f(Y) \neq \emptyset \Rightarrow \\
f(X)=f(Y) \cap X\end{array}$ \\
\hline & $\begin{array}{c}\left(\log =^{\prime}\right) \\
\operatorname{Con}\left(\overline{\overline{T^{\prime}}} \cup T\right) \Rightarrow \\
\overline{\overline{T \cup T^{\prime}}}=\overline{\overline{\overline{T^{\prime}}} \cup T}\end{array}$ & $\begin{array}{c}\left(\mu=^{\prime}\right) \\
f(Y) \cap X \neq \emptyset \Rightarrow \\
f(Y \cap X)=f(Y) \cap X\end{array}$ \\
\hline & $\begin{array}{c}(\log \|) \\
\overline{\overline{T \vee T^{\prime}}} \text { is one of } \\
\overline{\bar{T}} \text {, or } \overline{\overline{T^{\prime}}} \text {, or } \overline{\bar{T}} \cap \overline{\overline{T^{\prime}}}(\text { by }(\mathrm{CCL}))\end{array}$ & $\begin{array}{c}(\mu \|) \\
f(X \cup Y) \text { is one of } \\
f(X), f(Y) \text { or } f(X) \cup f(Y)\end{array}$ \\
\hline & $\begin{array}{c}(\log \cup) \\
\operatorname{Con}\left(\overline{\overline{T^{\prime}}} \cup T\right), \neg \operatorname{Con}\left(\overline{\overline{T^{\prime}}} \cup \overline{\bar{T}}\right) \Rightarrow \\
\neg \operatorname{Con}\left(\overline{\overline{T \vee T^{\prime}}} \cup T^{\prime}\right)\end{array}$ & $\begin{array}{c}(\mu \cup) \\
f(Y) \cap(X-f(X)) \neq \emptyset \Rightarrow \\
f(X \cup Y) \cap Y=\emptyset\end{array}$ \\
\hline & 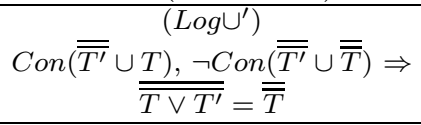 & $\begin{array}{c}\left(\mu \cup^{\prime}\right) \\
f(Y) \cap(X-f(X)) \neq \emptyset \Rightarrow \\
f(X \cup Y)=f(X)\end{array}$ \\
\hline & & $\begin{array}{c}(\mu \in) \\
a \in X-f(X) \Rightarrow \\
\exists b \in X . a \notin f(\{a, b\})\end{array}$ \\
\hline
\end{tabular}

$(P R)$ is also called infinite conditionalization - we choose the name for its central role for preferential structures $(P R)$ or $(\mu P R)$.

The system of rules $(A N D)(O R)(L L E)(R W)(S C)(C P)(C M)(C U M)$ is also called system $P$ (for preferential), adding (Rat $M)$ gives the system $R$ (for rationality or rankedness).

Roughly: Smooth preferential structures generate logics satisfying system $P$, ranked structures logics satisfying system $R$.

A logic satisfying $(R E F),(\operatorname{Res} M)$, and $(C U T)$ is called a consequence relation.

$(L L E)$ and $(C C L)$ will hold automatically, whenever we work with model sets.

$(A N D)$ is obviously closely related to filters, and corresponds to closure under finite intersections. $(R W)$ corresponds to upward closure of filters.

More precisely, validity of both depend on the definition, and the direction we consider.

Given $f$ and $(\mu \subseteq), f(X) \subseteq X$ generates a pricipal filter: $\left\{X^{\prime} \subseteq X: f(X) \subseteq X^{\prime}\right\}$, with the definition: If $X=M(T)$, then $T \sim \phi$ iff $f(X) \subseteq M(\phi)$. Validity of $(A N D)$ and $(R W)$ are then trivial.

Conversely, we can define for $X=M(T)$ 
$\mathcal{X}:=\left\{X^{\prime} \subseteq X: \exists \phi\left(X^{\prime}=X \cap M(\phi)\right.\right.$ and $\left.\left.T \sim \phi\right)\right\}$.

$(A N D)$ then makes $\mathcal{X}$ closed under finite intersections, $(R W)$ makes $\mathcal{X}$ upward closed. This is in the infinite case usually not yet a filter, as not all subsets of $X$ need to be definable this way. In this case, we complete $\mathcal{X}$ by adding all $X^{\prime \prime}$ such that there is $X^{\prime} \subseteq X^{\prime \prime} \subseteq X, X^{\prime} \in \mathcal{X}$.

Alternatively, we can define

$\mathcal{X}:=\left\{X^{\prime} \subseteq X: \bigcap\{X \cap M(\phi): T \sim \phi\} \subseteq X^{\prime}\right\}$.

$(S C)$ corresponds to the choice of a subset.

$(C P)$ is somewhat delicate, as it presupposes that the chosen model set is non-empty. This might fail in the presence of ever better choices, without ideal ones; the problem is addressed by the limit versions.

$(P R)$ is an infinitary version of one half of the deduction theorem: Let $T$ stand for $\phi, T^{\prime}$ for $\psi$, and $\phi \wedge \psi \sim \sigma$, so $\phi \sim \psi \rightarrow \sigma$, but $(\psi \rightarrow \sigma) \wedge \psi \vdash \sigma$.

$(C U M)$ (whose most interesting half in our context is $(C M)$ ) may best be seen as normal use of lemmas: We have worked hard and found some lemmas. Now we can take a rest, and come back again with our new lemmas. Adding them to the axioms will neither add new theorems, nor prevent old ones to hold.

\section{Fact 3.2}

This table is to be read as follows: If the left hand side holds for some function $f: \mathcal{Y} \rightarrow \mathcal{P}(U)$, and the auxiliary properties noted in the middle also hold for $f$ or $\mathcal{Y}$, then the right hand side will hold, too and conversely. 


\begin{tabular}{|c|c|c|c|}
\hline \multicolumn{4}{|c|}{ Basics } \\
\hline$(1.1)$ & \multirow[t]{2}{*}{$(\mu P R)$} & $\Rightarrow(\cap)+(\mu \subseteq)$ & \multirow[t]{2}{*}{$\left(\mu P R^{\prime}\right)$} \\
\hline$(1.2)$ & & $\Leftarrow$ & \\
\hline$(2.1)$ & \multirow[t]{2}{*}{$(\mu P R)$} & $\Rightarrow(\mu \subseteq)$ & \multirow[t]{2}{*}{$(\mu O R)$} \\
\hline$(2.2)$ & & $\begin{array}{l}\Leftarrow(\mu \subseteq)+\text { closure } \\
\text { under set difference }\end{array}$ & \\
\hline$(3)$ & $(\mu P R)$ & $\Rightarrow$ & $(\mu C U T)$ \\
\hline (4) & $\begin{array}{c}(\mu \subseteq)+(\mu \subseteq \supseteq)+(\mu C U M)+ \\
(\mu \text { Rat } M)+(\cap)\end{array}$ & $\not \Rightarrow$ & $(\mu P R)$ \\
\hline \multicolumn{4}{|c|}{ Cumulativity } \\
\hline$(5.1)$ & \multirow[t]{2}{*}{$(\mu C M)$} & $\Rightarrow(\cap)+(\mu \subseteq)$ & \multirow[t]{2}{*}{$(\mu \operatorname{Res} M)$} \\
\hline$(5.2)$ & & $\Leftarrow$ (infin.) & \\
\hline$(6)$ & $(\mu C M)+(\mu C U T)$ & $\Leftrightarrow$ & $(\mu C U M)$ \\
\hline$(7)$ & $(\mu \subseteq)+(\mu \subseteq \supseteq)$ & $\Rightarrow$ & $(\mu C U M)$ \\
\hline$(8)$ & $(\mu \subseteq)+(\mu C U M)+(\cap)$ & $\Rightarrow$ & $(\mu \subseteq \supseteq)$ \\
\hline$(9)$ & $(\mu \subseteq)+(\mu C U M)$ & $\not \Rightarrow$ & $(\mu \subseteq \supseteq)$ \\
\hline \multicolumn{4}{|c|}{ Rationality } \\
\hline$(10)$ & $(\mu \operatorname{Rat} M)+(\mu P R)$ & $\Rightarrow$ & $(\mu=)$ \\
\hline$(11)$ & $(\mu=)$ & $\Rightarrow$ & $(\mu P R)$ \\
\hline$(12.1)$ & \multirow[t]{2}{*}{$(\mu=)$} & $\Rightarrow(\cap)+(\mu \subseteq)$ & \multirow[t]{2}{*}{$\left(\mu=^{\prime}\right)$} \\
\hline$(12.2)$ & & $\Leftarrow$ & \\
\hline$(13)$ & $(\mu \subseteq),(\mu=)$ & $\Rightarrow(\cup)$ & $(\mu \cup)$, \\
\hline$(14)$ & $(\mu \subseteq),(\mu \emptyset),(\mu=)$ & $\Rightarrow(\cup)$ & $(\mu \|),\left(\mu \cup^{\prime}\right),(\mu C U M)$, \\
\hline$(15)$ & $(\mu \subseteq)+(\mu \|)$ & $\Rightarrow \mathcal{Y}$ closed under set difference & $(\mu=)$ \\
\hline$(16)$ & $\begin{array}{c}(\mu \|)+(\mu \in)+(\mu P R)+ \\
(\mu \subseteq)\end{array}$ & $\Rightarrow(\cup)+\mathcal{Y}$ contains singletons & $(\mu=)$, \\
\hline$(17)$ & $(\mu C U M)+(\mu=)$ & $\Rightarrow(\cup)+\mathcal{Y}$ contains singletons & $(\mu \in)$, \\
\hline$(18)$ & $(\mu C U M)+(\mu=)+(\mu \subseteq)$ & $\Rightarrow(\cup)$ & $(\mu \|)$, \\
\hline$(19)$ & $(\mu P R)+(\mu C U M)+(\mu \|)$ & $\Rightarrow$ sufficient, e.g. true in $\boldsymbol{D}_{\mathcal{L}}$ & $(\mu=)$. \\
\hline$(20)$ & $(\mu \subseteq)+(\mu P R)+(\mu=)$ & $\not \Rightarrow$ & $(\mu \|)$, \\
\hline$(21)$ & $(\mu \subseteq)+(\mu P R)+(\mu \|)$ & $\begin{array}{l}\nRightarrow \text { (without closure } \\
\text { under set difference), }\end{array}$ & $(\mu=)$ \\
\hline$(22)$ & $\begin{array}{c}(\mu \subseteq)+(\mu P R)+(\mu \|)+ \\
(\mu=)+(\mu \cup)\end{array}$ & $\not \Rightarrow$ & $\begin{array}{c}(\mu \in) \\
\text { (thus not representability } \\
\text { by ranked structures) }\end{array}$ \\
\hline
\end{tabular}

\section{Proposition 3.3}

The following table is to be read as follows:

Let a logic $\sim$ satisfies $(L L E)$ and $(C C L)$, and define a function $f: \boldsymbol{D}_{\mathcal{L}} \rightarrow \boldsymbol{D}_{\mathcal{L}}$ by $f(M(T)):=M(\overline{\bar{T}})$. Then $f$ is well defined, satisfies $(\mu d p)$, and $\overline{\bar{T}}=T h(f(M(T)))$.

If $\sim$ satisfies a rule in the left hand side, then - provided the additional properties noted in the middle for $\Rightarrow$ hold, too - $f$ will satisfy the property in the right hand side.

Conversely, if $f: \mathcal{Y} \rightarrow \mathcal{P}\left(M_{\mathcal{L}}\right)$ is a function, with $\boldsymbol{D}_{\mathcal{L}} \subseteq \mathcal{Y}$, and we define a logic $\sim$ by $\overline{\bar{T}}:=T h(f(M(T)))$, then $\sim$ satisfies $(L L E)$ and $(C C L)$. If $f$ satisfies $(\mu d p)$, then $f(M(T))=M(\overline{\bar{T}})$.

If $f$ satisfies a property in the right hand side, then - provided the additional properties noted in the middle for $\Leftarrow$ hold, too - $\sim$ will satisfy the property in the left hand side.

If "formula" is noted in the table, this means that, if one of the theories (the one named the same way in Definition 3.4 is equivalent to a formula, we can renounce on $(\mu d p)$. 


\begin{tabular}{|c|c|c|c|}
\hline \multicolumn{4}{|c|}{ Basics } \\
\hline$(1.1)$ & \multirow[t]{2}{*}{$(O R)$} & $\Rightarrow$ & \multirow[t]{2}{*}{$(\mu O R)$} \\
\hline$(1.2)$ & & $\Leftarrow$ & \\
\hline$(2.1)$ & \multirow[t]{2}{*}{$\overline{(\text { disjOR })}$} & $\Rightarrow$ & \multirow[t]{2}{*}{$\overline{(\mu d i s j O R)}$} \\
\hline$(2.2)$ & & $\Leftarrow$ & \\
\hline$(3.1)$ & \multirow[t]{2}{*}{$(w O R)$} & $\Rightarrow$ & \multirow[t]{2}{*}{$(\mu w O R)$} \\
\hline$(3.2)$ & & $\Leftarrow$ & \\
\hline$(4.1)$ & \multirow[t]{2}{*}{$(S C)$} & $\Rightarrow$ & \multirow[t]{2}{*}{$(\mu \subseteq)$} \\
\hline$(4.2)$ & & $\Leftarrow$ & \\
\hline$(5.1)$ & \multirow[t]{2}{*}{$(C P)$} & $\Rightarrow$ & \multirow[t]{2}{*}{$(\mu \emptyset)$} \\
\hline$(5.2)$ & & $\Leftarrow$ & \\
\hline$(6.1)$ & \multirow[t]{4}{*}{$(P R)$} & $\Rightarrow$ & \multirow[t]{4}{*}{$(\mu P R)$} \\
\hline$(6.2)$ & & $\Leftarrow(\mu d p)+(\mu \subseteq)$ & \\
\hline$(6.3)$ & & $\notin$ without $(\mu d p)$ & \\
\hline$(6.4)$ & & $\begin{array}{c}\Leftarrow(\mu \subseteq) \\
T^{\prime} \text { a formula }\end{array}$ & \\
\hline$(6.5)$ & $(P R)$ & $\begin{array}{c}\Leftarrow \\
T^{\prime} \text { a formula }\end{array}$ & $\left(\mu P R^{\prime}\right)$ \\
\hline$(7.1)$ & \multirow[t]{2}{*}{$(C U T)$} & $\Rightarrow$ & \multirow[t]{2}{*}{$(\mu C U T)$} \\
\hline$(7.2)$ & & $\Leftarrow$ & \\
\hline \multicolumn{4}{|c|}{ Cumulativity } \\
\hline$(8.1)$ & \multirow[t]{2}{*}{$(C M)$} & $\Rightarrow$ & \multirow[t]{2}{*}{$(\mu C M)$} \\
\hline$(8.2)$ & & $\Leftarrow$ & \\
\hline$(9.1)$ & \multirow[t]{2}{*}{$(\operatorname{Res} M)$} & $\Rightarrow$ & \multirow[t]{2}{*}{$\overline{(\mu \operatorname{Res} M)}$} \\
\hline$(9.2)$ & & $\Leftarrow$ & \\
\hline$(10.1)$ & \multirow[t]{2}{*}{$(\subseteq \supseteq)$} & $\Rightarrow$ & \multirow[t]{2}{*}{$(\mu \subseteq \supseteq)$} \\
\hline$(10.2)$ & & $\Leftarrow$ & \\
\hline$(11.1)$ & \multirow[t]{2}{*}{$(C U M)$} & $\Rightarrow$ & $(\mu C U M)$ \\
\hline$(11.2)$ & & $\Leftarrow$ & \\
\hline & & Rationality & \\
\hline$(12.1)$ & $(\operatorname{Rat} M)$ & $\Rightarrow$ & $(\mu \operatorname{RatM})$ \\
\hline$(12.2)$ & & $\Leftarrow(\mu d p)$ & \\
\hline$(12.3)$ & & $\notin$ without $(\mu d p)$ & \\
\hline$(12.4)$ & & $\begin{array}{c}\Leftarrow \\
T \text { a formula }\end{array}$ & \\
\hline$(13.1)$ & $(\operatorname{Rat} M=)$ & $\Rightarrow$ & $(\mu=)$ \\
\hline$(13.2)$ & & $\Leftarrow(\mu d p)$ & \\
\hline$(13.3)$ & & $\notin$ without $(\mu d p)$ & \\
\hline$(13.4)$ & & $\begin{array}{c}\Leftarrow \\
T \text { a formula }\end{array}$ & \\
\hline$(14.1)$ & $\left(\log =^{\prime}\right)$ & $\Rightarrow$ & $\left(\mu=^{\prime}\right)$ \\
\hline$(14.2)$ & & $\Leftarrow(\mu d p)$ & \\
\hline$(14.3)$ & & $\notin=$ without $(\mu d p)$ & \\
\hline$(14.4)$ & & $\Leftarrow T$ a formula & \\
\hline$(15.1)$ & $(\log \|)$ & $\Rightarrow$ & $(\mu \|)$ \\
\hline$(15.2)$ & & $\Leftarrow$ & \\
\hline$(16.1)$ & $(\log \cup)$ & $\Rightarrow(\mu \subseteq)+(\mu=)$ & $(\mu \cup)$ \\
\hline$(16.2)$ & & $\Leftarrow(\mu d p)$ & \\
\hline$(16.3)$ & & $\notin$ without $(\mu d p)$ & \\
\hline$(17.1)$ & $\left(\log \cup^{\prime}\right)$ & $\Rightarrow(\mu \subseteq)+(\mu=)$ & $\left(\mu \cup^{\prime}\right)$ \\
\hline$(17.2)$ & & $\Leftarrow(\mu d p)$ & \\
\hline$(17.3)$ & & $\notin$ without $(\mu d p)$ & \\
\hline
\end{tabular}




\section{Definition 3.5}

Fix $U \neq \emptyset$, and consider arbitrary $X$. Note that this $X$ has not necessarily anything to do with $U$, or $\mathcal{U}$ below. Thus, the functions $\mu_{\mathcal{M}}$ below are in principle functions from $V$ to $V$ - where $V$ is the set theoretical universe we work in.

(A) Preferential models or structures.

(1) The version without copies:

A pair $\mathcal{M}:=<U, \prec>$ with $U$ an arbitrary set, and $\prec$ an arbitrary binary relation is called a preferential model or structure.

(2) The version with copies:

A pair $\mathcal{M}:=<\mathcal{U}, \prec>$ with $\mathcal{U}$ an arbitrary set of pairs, and $\prec$ an arbitrary binary relation is called a preferential model or structure.

If $\langle x, i>\in \mathcal{U}$, then $x$ is intended to be an element of $U$, and $i$ the index of the copy.

We sometimes also need copies of the relation $\prec$, we will then replace $\prec$ by one or several arrows $\alpha$ attacking non-minimal elements, e.g. $x \prec y$ will be written $\alpha: x \rightarrow y,\langle x, i>\prec<y, i>$ will be written $\alpha:\langle x, i>\rightarrow<y, i>$, and finally we might have $\langle\alpha, k>: x \rightarrow y$ and $\langle\alpha, k>:<x, i>\rightarrow<y, i>$, etc.

(B) Minimal elements, the functions $\mu_{\mathcal{M}}$

(1) The version without copies:

Let $\mathcal{M}:=<U, \prec>$, and define

$\mu_{\mathcal{M}}(X):=\left\{x \in X: x \in U \wedge \neg \exists x^{\prime} \in X \cap U \cdot x^{\prime} \prec x\right\}$.

$\mu_{\mathcal{M}}(X)$ is called the set of minimal elements of $X$ (in $\left.\mathcal{M}\right)$.

(2) The version with copies:

Let $\mathcal{M}:=<\mathcal{U}, \prec>$ be as above. Define

$\mu_{\mathcal{M}}(X):=\left\{x \in X: \exists<x, i>\in \mathcal{U} . \neg \exists<x^{\prime}, i^{\prime}>\in \mathcal{U}\left(x^{\prime} \in X \wedge<x^{\prime}, i^{\prime}>^{\prime} \prec<x, i>\right)\right\}$.

Again, by abuse of language, we say that $\mu_{\mathcal{M}}(X)$ is the set of minimal elements of $X$ in the structure. If the context is clear, we will also write just $\mu$.

We sometimes say that $\langle x, i\rangle$ "kills" or "minimizes" $<y, j\rangle$ if $<x, i>\prec<y, j\rangle$. By abuse of language we also say a set $X$ kills or minimizes a set $Y$ if for all $<y, j>\in \mathcal{U}, y \in Y$ there is $\langle x, i>\in \mathcal{U}$, $x \in X$ s.t. $\langle x, i>\prec<y, j>$.

$\mathcal{M}$ is also called injective or 1 -copy, iff there is always at most one copy $\langle x, i\rangle$ for each $x$. Note that the existence of copies corresponds to a non-injective labelling function - as is often used in nonclassical logic, e.g. modal logic.

We say that $\mathcal{M}$ is transitive, irreflexive, etc., iff $\prec$ is.

Note that $\mu(X)$ might well be empty, even if $X$ is not.

\section{Definition 3.6}

We define the consequence relation of a preferential structure for a given propositional language $\mathcal{L}$.

(A)

(1) If $m$ is a classical model of a language $\mathcal{L}$, we say by abuse of language

$<m, i>\models \phi$ iff $m \models \phi$,

and if $X$ is a set of such pairs, that 
$X \models \phi$ iff for all $<m, i>\in X m \models \phi$.

(2) If $\mathcal{M}$ is a preferential structure, and $X$ is a set of $\mathcal{L}$-models for a classical propositional language $\mathcal{L}$, or a set of pairs $\langle m, i\rangle$, where the $m$ are such models, we call $\mathcal{M}$ a classical preferential structure or model.

(B)

Validity in a preferential structure, or the semantical consequence relation defined by such a structure:

Let $\mathcal{M}$ be as above.

We define:

$T \models \mathcal{M} \phi$ iff $\mu_{\mathcal{M}}(M(T)) \models \phi$, i.e. $\mu_{\mathcal{M}}(M(T)) \subseteq M(\phi)$.

$\mathcal{M}$ will be called definability preserving iff for all $X \in \boldsymbol{D}_{\mathcal{L}} \mu_{\mathcal{M}}(X) \in \boldsymbol{D}_{\mathcal{L}}$.

As $\mu_{\mathcal{M}}$ is defined on $\boldsymbol{D}_{\mathcal{L}}$, but need by no means always result in some new definable set, this is (and reveals itself as a quite strong) additional property.

\section{Definition 3.7}

Let $\mathcal{Y} \subseteq \mathcal{P}(U)$. (In applications to logic, $\mathcal{Y}$ will be $\boldsymbol{D}_{\mathcal{L}}$.)

A preferential structure $\mathcal{M}$ is called $\mathcal{Y}$-smooth iff in every $X \in \mathcal{Y}$ every element $x \in X$ is either minimal in $X$ or above an element, which is minimal in $X$. More precisely:

(1) The version without copies:

If $x \in X \in \mathcal{Y}$, then either $x \in \mu(X)$ or there is $x^{\prime} \in \mu(X) \cdot x^{\prime} \prec x$.

(2) The version with copies:

If $x \in X \in \mathcal{Y}$, and $<x, i>\in \mathcal{U}$, then either there is no $<x^{\prime}, i^{\prime}>\in \mathcal{U}, x^{\prime} \in X,<x^{\prime}, i^{\prime}>\prec<x, i>$ or there is $<x^{\prime}, i^{\prime}>\in \mathcal{U},<x^{\prime}, i^{\prime}>\prec<x, i>, x^{\prime} \in X$, s.t. there is no $<x^{\prime \prime}, i^{\prime \prime}>\in \mathcal{U}, x^{\prime \prime} \in X$, with $<x^{\prime \prime}, i^{\prime \prime}>\prec<x^{\prime}, i^{\prime}>$.

When considering the models of a language $\mathcal{L}, \mathcal{M}$ will be called smooth iff it is $\boldsymbol{D}_{\mathcal{L}}$ - $\operatorname{smooth} ; \boldsymbol{D}_{\mathcal{L}}$ is the default.

Obviously, the richer the set $\mathcal{Y}$ is, the stronger the condition $\mathcal{Y}$-smoothness will be.

The following table summarizes representation by not necessarily ranked preferential structures. The implications on the right are shown in Proposition 3.3 (going via the $\mu$-functions), those on the left are shown in the respective representation theorems.

\begin{tabular}{|c|c|c|c|c|}
\hline$\mu-$ function & & Pref.Structure & & Logic \\
\hline \multirow{3}{*}{$(\mu \subseteq)+(\mu P R)$} & $\Leftarrow$ & \multirow[t]{3}{*}{ general } & $\Rightarrow(\mu d p)$ & \multirow{3}{*}{$(L L E)+(R W)+(S C)+(P R)$} \\
\hline & $\Rightarrow$ & & $\Leftarrow$ & \\
\hline & & & $\nexists$ without $(\mu d p)$ & \\
\hline \multirow[t]{2}{*}{$(\mu \subseteq)+(\mu P R)$} & $\Leftarrow$ & \multirow[t]{2}{*}{ transitive } & $\Rightarrow(\mu d p)$ & \multirow{2}{*}{$(L L E)+(R W)+(S C)+(P R)$} \\
\hline & $\Rightarrow$ & & $\stackrel{\Leftarrow}{\Leftarrow}$ & \\
\hline \multirow[t]{3}{*}{$(\mu \subseteq)+(\mu P R)+(\mu C U M)$} & $\Leftarrow$ & \multirow[t]{3}{*}{ smooth } & $\Rightarrow(\mu d p)$ & \multirow{3}{*}{$\begin{array}{c}(L L E)+(R W)+(S C)+(P R)+ \\
(C U M)\end{array}$} \\
\hline & $\Rightarrow(\cup)$ & & $\Leftarrow(U)$ & \\
\hline & & & $\nexists$ without $(\mu d p)$ & \\
\hline \multirow[t]{2}{*}{$(\mu \subseteq)+(\mu P R)+(\mu C U M)$} & $\Leftarrow$ & \multirow[t]{2}{*}{ smooth+transitive } & $\Rightarrow(\mu d p)$ & \multirow[t]{2}{*}{$\begin{array}{c}(L L E)+(R W)+(S C)+(P R)+ \\
(C U M)\end{array}$} \\
\hline & $\Rightarrow(\cup)$ & & $\Leftarrow(\cup)$ & \\
\hline
\end{tabular}




\subsection{Generalized preferential structures}

\subsubsection{Introduction}

\section{Comment 3.1}

A counterargument to $\alpha$ is NOT an argument for $\neg \alpha$ (this is asking for too much), but just showing one case where $\neg \alpha$ holds. In preferential structures, an argument for $\alpha$ is a set of level 1 arrows, eliminating $\neg \alpha$-models. A counterargument is one level 2 arrow, attacking one such level 1 arrow.

Of course, when we have copies, we may need many successful attacks, on all copies, to achieve the goal. As we may have copies of level 1 arrows, we may need many level 2 arrows to destroy them all.

\section{Definition 3.8}

An IBR is called a generalized preferential structure iff the origins of all arrows are points. We will usually write $x, y$ etc. for points, $\alpha, \beta$ etc. for arrows.

\section{Definition 3.9}

Consider a generalized preferential structure $\mathcal{X}$.

(1) Level $n$ arrow:

Definition by upward induction.

If $\alpha: x \rightarrow y, x, y$ are points, then $\alpha$ is a level 1 arrow.

If $\alpha: x \rightarrow \beta, x$ is a point, $\beta$ a level $n$ arrow, then $\alpha$ is a level $n+1$ arrow. $(o(\alpha)$ is the origin, $d(\alpha)$ is the destination of $\alpha$.)

$\lambda(\alpha)$ will denote the level of $\alpha$.

(2) Level $n$ structure:

$\mathcal{X}$ is a level $n$ structure iff all arrows in $\mathcal{X}$ are at most level $n$ arrows.

We consider here only structures of some arbitrary but finite level $n$.

(3) We define for an arrow $\alpha$ by induction $O(\alpha)$ and $D(\alpha)$.

If $\lambda(\alpha)=1$, then $O(\alpha):=\{o(\alpha)\}, D(\alpha):=\{d(\alpha)\}$.

If $\alpha: x \rightarrow \beta$, then $D(\alpha):=D(\beta)$, and $O(\alpha):=\{x\} \cup O(\beta)$.

Thus, for example, if $\alpha: x \rightarrow y, \beta: z \rightarrow \alpha$, then $O(\beta):=\{x, z\}, D(\beta)=\{y\}$.

We will not consider here diagrams with arbitrarily high levels. One reason is that diagrams like the following will have an unclear meaning:

\section{Example 3.1}

$<\alpha, 1>: x \rightarrow y$,

$<\alpha, n+1>: x \rightarrow<\alpha, n>(n \in \omega)$.

Is $y \in \mu(X)$ ?

\section{Definition 3.10}

Let $\mathcal{X}$ be a generalized preferential structure of (finite) level $n$.

We define (by downward induction): 
(1) Valid $X-$ to $-Y$ arrow:

Let $X, Y \subseteq \boldsymbol{P}(\mathcal{X})$.

$\alpha \in \boldsymbol{A}(\mathcal{X})$ is a valid $X-$ to $-Y$ arrow iff

(1.1) $O(\alpha) \subseteq X, D(\alpha) \subseteq Y$,

(1.2) $\forall \beta: x^{\prime} \rightarrow \alpha .\left(x^{\prime} \in X \Rightarrow \exists \gamma: x^{\prime \prime} \rightarrow \beta .(\gamma\right.$ is a valid $X-t o-Y$ arrow $\left.)\right)$.

We will also say that $\alpha$ is a valid arrow in $X$, or just valid in $X$, iff $\alpha$ is a valid $X-$ to $-X$ arrow.

(2) Valid $X \Rightarrow Y$ arrow:

Let $X \subseteq Y \subseteq \boldsymbol{P}(\mathcal{X})$.

$\alpha \in \boldsymbol{A}(\mathcal{X})$ is a valid $X \Rightarrow Y$ arrow iff

(2.1) $o(\alpha) \in X, O(\alpha) \subseteq Y, D(\alpha) \subseteq Y$,

(2.2) $\forall \beta: x^{\prime} \rightarrow \alpha .\left(x^{\prime} \in Y \Rightarrow \exists \gamma: x^{\prime \prime} \rightarrow \beta .(\gamma\right.$ is a valid $X \Rightarrow Y$ arrow $\left.)\right)$.

(Note that in particular $o(\gamma) \in X$, and that $o(\beta)$ need not be in $X$, but can be in the bigger $Y$.)

\section{Fact 3.4}

(1) If $\alpha$ is a valid $X \Rightarrow Y$ arrow, then $\alpha$ is a valid $Y-t o-Y$ arrow.

(2) If $X \subseteq X^{\prime} \subseteq Y^{\prime} \subseteq Y \subseteq \boldsymbol{P}(\mathcal{X})$ and $\alpha \in \boldsymbol{A}(\mathcal{X})$ is a valid $X \Rightarrow Y$ arrow, and $O(\alpha) \subseteq Y^{\prime}, D(\alpha) \subseteq Y^{\prime}$, then $\alpha$ is a valid $X^{\prime} \Rightarrow Y^{\prime}$ arrow.

\section{Proof Fact Higher-Validity}

Let $\alpha$ be a valid $X \Rightarrow Y$ arrow. We show (1) and (2) together by downward induction (both are trivial). By prerequisite $o(\alpha) \in X \subseteq X^{\prime}, O(\alpha) \subseteq Y^{\prime} \subseteq Y, D(\alpha) \subseteq Y^{\prime} \subseteq Y$.

Case 1: $\lambda(\alpha)=n$. So $\alpha$ is a valid $X^{\prime} \Rightarrow Y^{\prime}$ arrow, and a valid $Y-$ to $-Y$ arrow.

Case 2: $\lambda(\alpha)=n-1$. So there is no $\beta: x^{\prime} \rightarrow \alpha, y \in Y$, so $\alpha$ is a valid $Y-t o-Y$ arrow. By $Y^{\prime} \subseteq Y \alpha$ is a valid $X^{\prime} \Rightarrow Y^{\prime}$ arrow.

Case 3: Let the result be shown down to $m, n>m>1$, let $\lambda(\alpha)=m-1$. So $\forall \beta: x^{\prime} \rightarrow \alpha\left(x^{\prime} \in Y \Rightarrow\right.$ $\exists \gamma: x^{\prime \prime} \rightarrow \beta\left(x^{\prime \prime} \in X\right.$ and $\gamma$ is a valid $X \Rightarrow Y$ arrow $\left.)\right)$. By induction hypothesis $\gamma$ is a valid $Y-t o-Y$ arrow, and a valid $X^{\prime} \Rightarrow Y^{\prime}$ arrow. So $\alpha$ is a valid $Y-t o-Y$ arrow, and by $Y^{\prime} \subseteq Y, \alpha$ is a valid $X^{\prime} \Rightarrow Y^{\prime}$ arrow.

\section{Definition 3.11}

Let $\mathcal{X}$ be a generalized preferential structure of level $n, X \subseteq \boldsymbol{P}(\mathcal{X})$.

$\mu(X):=\left\{x \in X: \exists<x, i>. \neg \exists\right.$ valid $X-$ to $-X$ arrow $\left.\alpha: x^{\prime} \rightarrow<x, i>\right\}$.

\section{Comment 3.2}

The purpose of smoothness is to guarantee cumulativity. Smoothness achieves Cumulativity by mirroring all information present in $X$ also in $\mu(X)$. Closer inspection shows that smoothness does more than necessary. This is visible when there are copies (or, equivalently, non-injective labelling functions). Suppose we have two copies of $x \in X,\left\langle x, i>\right.$ and $\left\langle x, i^{\prime}\right\rangle$, and there is $y \in X, \alpha:\langle y, j\rangle \rightarrow\langle x, i\rangle$, 
but there is no $\alpha^{\prime}:<y^{\prime}, j^{\prime}>\rightarrow<x, i^{\prime}>, y^{\prime} \in X$. Then $\alpha:<y, j>\rightarrow<x, i>$ is irrelevant, as $x \in \mu(X)$ anyhow. So mirroring $\alpha:<y, j>\rightarrow<x, i>$ in $\mu(X)$ is not necessary, i.e. it is not necessary to have some $\alpha^{\prime}:<y^{\prime}, j^{\prime}>\rightarrow<x, i>, y^{\prime} \in \mu(X)$.

On the other hand, Example 3.3 shows that, if we want smooth structures to correspond to the property $(\mu C U M)$, we need at least some valid arrows from $\mu(X)$ also for higher level arrows. This "some" is made precise (essentially) in Definition 3.12.

From a more philosophical point of view, when we see the (inverted) arrows of preferential structures as attacks on non-minimal elements, then we should see smooth structures as always having attacks also from valid (minimal) elements. So, in general structures, also attacks from non-valid elements are valid, in smooth structures we always also have attacks from valid elements.

The analogon to usual smooth structures, on level 2, is then that any successfully attacked level 1 arrow is also attacked from a minimal point.

\section{Definition 3.12}

Let $\mathcal{X}$ be a generalized preferential structure.

$X \sqsubseteq X^{\prime}$ iff

(1) $X \subseteq X^{\prime} \subseteq \boldsymbol{P}(\mathcal{X})$

(2) $\forall x \in X^{\prime}-X \forall<x, i>\exists \alpha: x^{\prime} \rightarrow<x, i>$ ( $\alpha$ is a valid $X \Rightarrow X^{\prime}$ arrow),

(3) $\forall x \in X \exists<x, i>$

$$
\left(\forall \alpha: x^{\prime} \rightarrow<x, i>\left(x^{\prime} \in X^{\prime} \Rightarrow \exists \beta: x^{\prime \prime} \rightarrow \alpha .\left(\beta \text { is a valid } X \Rightarrow X^{\prime} \text { arrow }\right)\right)\right) .
$$

Note that (3) is not simply the negation of (2):

Consider a level 1 structure. Thus all level 1 arrows are valid, but the source of the arrows must not be neglected.

(2) reads now: $\forall x \in X^{\prime}-X \forall<x, i>\exists \alpha: x^{\prime} \rightarrow<x, i>. x^{\prime} \in X$

(3) reads: $\forall x \in X \exists<x, i>\neg \exists \alpha: x^{\prime} \rightarrow<x, i>. x^{\prime} \in X^{\prime}$

This is intended: intuitively, $X=\mu\left(X^{\prime}\right)$, and minimal elements must not be attacked at all, but nonminimals must be attacked from $X$ - which is a modified version of smoothness.

\section{Remark 3.5}

We note the special case of Definition 3.12 for level 3 structures, as it will be used later. We also write it immediately for the intended case $\mu(X) \sqsubseteq X$, and explicitly with copies.

$x \in \mu(X)$ iff

(1) $\exists<x, i>\forall<\alpha, k>:<y, j>\rightarrow<x, i>$

$$
\begin{aligned}
& \left(y \in X \rightarrow \exists<\beta^{\prime}, l^{\prime}>:<z^{\prime}, m^{\prime}>\rightarrow<\alpha, k>.\right. \\
& \left.\quad\left(z^{\prime} \in \mu(X) \wedge \neg \exists<\gamma^{\prime}, n^{\prime}>:<u^{\prime}, p^{\prime}>\rightarrow<\beta^{\prime}, l^{\prime}>. u^{\prime} \in X\right)\right)
\end{aligned}
$$

See Diagram 3.1 .

$x \in X-\mu(X)$ iff

(2) $\forall<x, i>\exists<\alpha^{\prime}, k^{\prime}>:<y^{\prime}, j^{\prime}>\rightarrow<x, i>$

$\left(y^{\prime} \in \mu(X) \wedge\right.$

(a) $\neg \exists<\beta^{\prime}, l^{\prime}>:<z^{\prime}, m^{\prime}>\rightarrow<\alpha^{\prime}, k^{\prime}>. z^{\prime} \in X$

or 
(b) $\forall<\beta^{\prime}, l^{\prime}>:<z^{\prime}, m^{\prime}>\rightarrow<\alpha^{\prime}, k^{\prime}>$

$\left.\left(z^{\prime} \in X \rightarrow \exists<\gamma^{\prime}, n^{\prime}>:<u^{\prime}, p^{\prime}>\rightarrow<\beta^{\prime}, l^{\prime}>. u^{\prime} \in \mu(X)\right)\right)$

See Diagram 3.2.

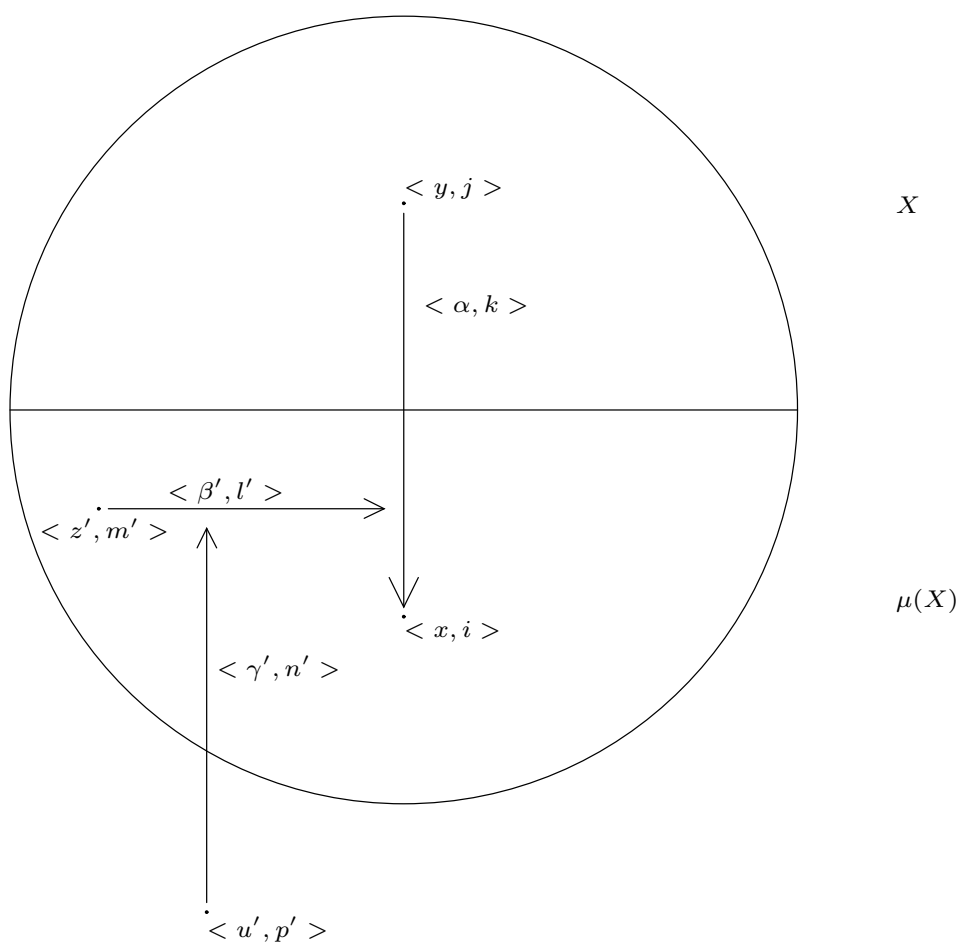

Case 3-1-2

Diagram 3.1 


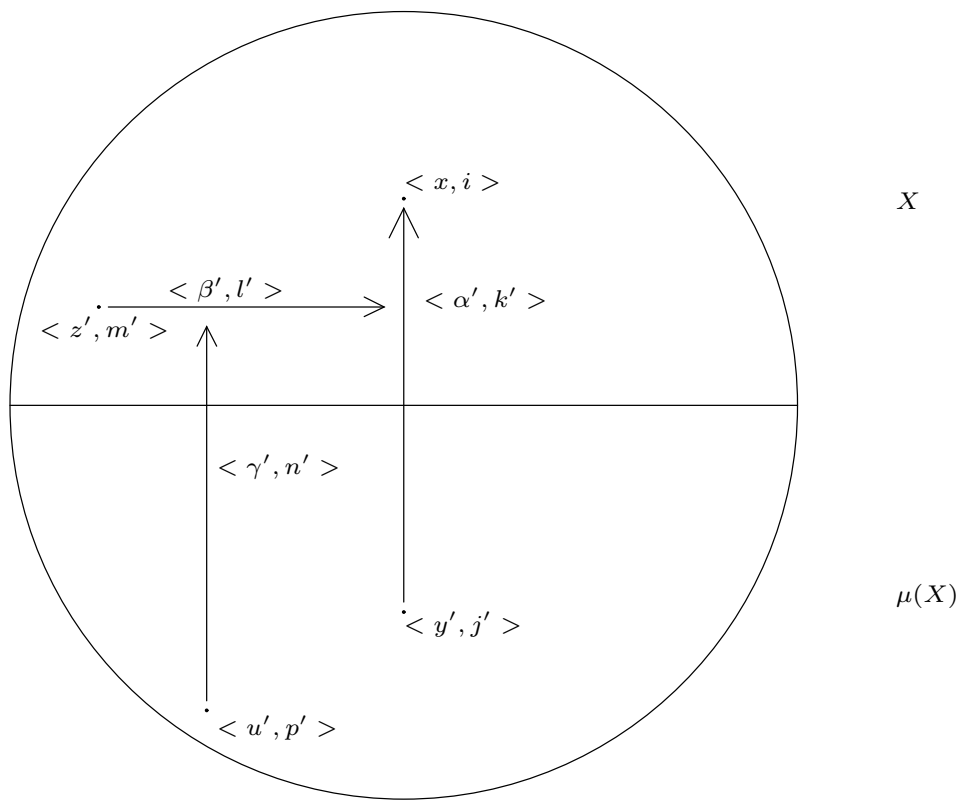

Diagram 3.2

Case 3-2

\section{Fact 3.6}

(1) If $X \sqsubseteq X^{\prime}$, then $X=\mu\left(X^{\prime}\right)$,

(2) $X \sqsubseteq X^{\prime}, X \subseteq X^{\prime \prime} \subseteq X^{\prime} \Rightarrow X \sqsubseteq X^{\prime \prime}$. (This corresponds to $(\mu C U M)$.)

(3) $X \sqsubseteq X^{\prime}, X \subseteq Y^{\prime}, Y \sqsubseteq Y^{\prime}, Y \subseteq X^{\prime} \Rightarrow X=Y$. (This corresponds to $(\mu \subseteq \supseteq$ ).)

Proof Fact $X-S u b-X^{\prime}$

\section{Proof}

(1) Trivial by Fact 3.4 (1).

(2)

We have to show

(a) $\forall x \in X^{\prime \prime}-X \forall<x, i>\exists \alpha: x^{\prime} \rightarrow<x, i>$ ( $\alpha$ is a valid $X \Rightarrow X^{\prime \prime}$ arrow), and

(b) $\forall x \in X \exists<x, i>\left(\forall \alpha: x^{\prime} \rightarrow<x, i>\left(x^{\prime} \in X^{\prime \prime} \Rightarrow \exists \beta: x^{\prime \prime} \rightarrow \alpha\right.\right.$.( $\beta$ is a valid $X \Rightarrow X^{\prime \prime}$ arrow $\left.\left.)\right)\right)$.

Both follow from the corresponding condition for $X \Rightarrow X^{\prime}$, the restriction of the universal quantifier, and Fact 3.4 (2).

(3) 
Let $x \in X-\mathrm{Y}$.

(a) By $x \in X \sqsubseteq X^{\prime}, \exists<x, i>$ s.t. $\left(\forall \alpha: x^{\prime} \rightarrow<x, i>\left(x^{\prime} \in X^{\prime} \Rightarrow \exists \beta: x^{\prime \prime} \rightarrow \alpha\right.\right.$. $\left(\beta\right.$ is a valid $X \Rightarrow X^{\prime}$ arrow))).

(b) By $x \notin Y \sqsubseteq \exists \alpha_{1}: x^{\prime} \rightarrow<x, i>\alpha_{1}$ is a valid $Y \Rightarrow Y^{\prime}$ arrow, in particular $x^{\prime} \in Y \subseteq X^{\prime}$. Moreover, $\lambda\left(\alpha_{1}\right)=1$.

So by (a) $\exists \beta_{2}: x^{\prime \prime} \rightarrow \alpha$. ( $\left(\beta_{2}\right.$ is a valid $X \Rightarrow X^{\prime}$ arrow), in particular $x^{\prime \prime} \in X \subseteq Y^{\prime}$, moreover $\lambda\left(\beta_{2}\right)=2$.

It follows by induction from the definition of valid $A \Rightarrow B$ arrows that

$\forall n \exists \alpha_{2 m+1}, \lambda\left(\alpha_{2 m+1}\right)=2 m+1, \alpha_{2 m+1}$ a valid $Y \Rightarrow Y^{\prime}$ arrow and

$\forall n \exists \beta_{2 m+2}, \lambda\left(\beta_{2 m+2}\right)=2 m+2, \beta_{2 m+2}$ a valid $X \Rightarrow X^{\prime}$ arrow,

which is impossible, as $\mathcal{X}$ is a structure of finite level.

\section{Definition 3.13}

Let $\mathcal{X}$ be a generalized preferential structure, $X \subseteq \boldsymbol{P}(\mathcal{X})$.

$\mathcal{X}$ is called totally smooth for $X$ iff

(1) $\forall \alpha: x \rightarrow y \in \boldsymbol{A}(\mathcal{X})\left(O(\alpha) \cup D(\alpha) \subseteq X \Rightarrow \exists \alpha^{\prime}: x^{\prime} \rightarrow y \cdot x^{\prime} \in \mu(X)\right)$

(2) if $\alpha$ is valid, then $\alpha^{\prime}$ must also be valid.

(y a point or an arrow).

If $\mathcal{Y} \subseteq \boldsymbol{P}(\mathcal{X})$, then $\mathcal{X}$ is called $\mathcal{Y}$-totally smooth iff for all $X \in \mathcal{Y} \mathcal{X}$ is totally smooth for $X$.

\section{Example 3.2}

$X:=\left\{\alpha: a \rightarrow b, \alpha^{\prime}: b \rightarrow c, \alpha^{\prime \prime}: a \rightarrow c, \beta: b \rightarrow \alpha^{\prime}\right\}$ is not totally smooth,

$X:=\left\{\alpha: a \rightarrow b, \alpha^{\prime}: b \rightarrow c, \alpha^{\prime \prime}: a \rightarrow c, \beta: b \rightarrow \alpha^{\prime}, \beta^{\prime}: a \rightarrow \alpha^{\prime}\right\}$ is totally smooth.

\section{Example 3.3}

Consider $\alpha^{\prime}: a \rightarrow b, \alpha^{\prime \prime}: b \rightarrow c, \alpha: a \rightarrow c, \beta: a \rightarrow \alpha$.

Then $\mu(\{a, b, c\})=\{a\}, \mu(\{a, c\})=\{a, c\}$. Thus, $(\mu C U M)$ does not hold in this structure. Note that there is no valid arrow from $\mu(\{a, b, c\})$ to $c$.

\section{Definition 3.14}

Let $\mathcal{X}$ be a generalized preferential structure, $X \subseteq \boldsymbol{P}(\mathcal{X})$.

$\mathcal{X}$ is called essentially smooth for $X$ iff $\mu(X) \sqsubseteq X$.

If $\mathcal{Y} \subseteq \boldsymbol{P}(\mathcal{X})$, then $\mathcal{X}$ is called $\mathcal{Y}$-essentially smooth iff for all $X \in \mathcal{Y} \mu(X) \sqsubseteq X$.

\section{Example 3.4}

It is easy to see that we can distinguish total and essential smoothness in richer structures, as the following Example shows:

We add an accessibility relation $R$, and consider only those models which are accessible. 
Let e.g. $a \rightarrow b \rightarrow<c, 0>,\langle c, 1>$, without transitivity. Thus, only $c$ has two copies. This structure is essentially smooth, but of course not totally so.

Let now mRa, mRb, $m R<c, 0>, m R<c, 1>, m^{\prime} R a, m^{\prime} R b, m^{\prime} R<c, 0>$.

Thus, seen from $m, \mu(\{a, b, c\})=\{a, c\}$, but seen from $m^{\prime}, \mu(\{a, b, c\})=\{a\}$, but $\mu(\{a, c\})=\{a, c\}$, contradicting (CUM).

\subsubsection{Results on not necessarily smooth structures}

\section{Example 3.5}

We show here $(\mu \subseteq)+(\mu \subseteq \supseteq)+(\mu C U M)+(\mu R a t M)+(\cap) \nRightarrow(\mu P R)$.

Let $U:=\{a, b, c\}$. Let $\mathcal{Y}=\mathcal{P}(U)$. So $(\cap)$ is trivially satisfied. Set $f(X):=X$ for all $X \subseteq U$ except for $f(\{a, b\})=\{b\}$. Obviously, this cannot be represented by a preferential structure and $(\mu P R)$ is false for $U$ and $\{a, b\}$. But it satisfies $(\mu \subseteq),(\mu C U M),(\mu R a t M) .(\mu \subseteq)$ is trivial. $(\mu C U M)$ : Let $f(X) \subseteq Y \subseteq X$. If $f(X)=X$, we are done. Consider $f(\{a, b\})=\{b\}$. If $\{b\} \subseteq Y \subseteq\{a, b\}$, then $f(Y)=\{b\}$, so we are done again. It is shown in Fact 3.2, (8) that $(\mu \subseteq \supseteq)$ follows. $(\mu R a t M)$ : Suppose $X \subseteq Y, X \cap f(Y) \neq \emptyset$, we have to show $f(X) \subseteq f(Y) \cap X$. If $f(Y)=Y$, the result holds by $X \subseteq Y$, so it does if $X=Y$. The only remaining case is $Y=\{a, b\}, X=\{b\}$, and the result holds again.

The idea to solve the representation problem illustrated by Example 3.5 is to use the points $c$ and $d$ as bases for counterarguments against $\alpha: b \rightarrow a-$ as is possible in IBRS. We do this now. We will obtain a representation for logics weaker than $P$ by generalized preferential structures.

\section{Definition 3.15}

NOETIG???

(1) Let $X$ be a diagram and $Y \subseteq X$. $Y$ is called a legal subdiagram of $X$ iff it can be obtained from $X$ and the set of points of $Y$ inductively as follows:

(1.1) all points of $Y$ are in $Y$

(1.2) if $a, b$ (b a point or arrow) are in $Y$, then so is any arrow from a to $b$, which is in $X$.

(These are all the objects in $Y$ ).

(2) If we work with copies of points, then we define: $Y$ is called a legal subdiagram of $X$ iff it can be obtained from $X$ and the set of points of $Y$ inductively as follows:

(2.1) if there is some $\left\langle y, i>\right.$ in $Y \subseteq X$, then all $\left.<y, i^{\prime}\right\rangle \in X$ are in $Y$ too,

(2.2) if $a, b$ (b a point or arrow) are in $Y$, then so is any arrow from a to $b$, which is in $X$.

(These are all the objects in $Y$ ). 
From now on, all subdiagrams will be legal.

We will now prove a representation theorem, but will make it more general than for preferential structures only. For this purpose, we will introduce some definitions first.

\section{Definition 3.16}

Let $\eta, \rho: \mathcal{Y} \rightarrow \mathcal{P}(U)$.

(1) If $\mathcal{X}$ is a simple structure:

$\mathcal{X}$ is called an attacking structure relative to $\eta$ representing $\rho$ iff $\rho(X)=\left\{x \in \eta(X)\right.$ : there is no valid $X-$ to $-\eta(X)$ arrow $\left.\alpha: x^{\prime} \rightarrow x\right\}$ for all $X \in \mathcal{Y}$.

(2) If $\mathcal{X}$ is a structure with copies:

$\mathcal{X}$ is called an attacking structure relative to $\eta$ representing $\rho$ iff $\rho(X)=\left\{x \in \eta(X)\right.$ : there is $<x, i>$ and no valid $X-$ to $-\eta(X)$ arrow $\left.\alpha:<x^{\prime}, i^{\prime}>\rightarrow<x, i>\right\}$ for all $X \in \mathcal{Y}$.

Obviously, in those cases $\rho(X) \subseteq \eta(X)$ for all $X \in \mathcal{Y}$.

Thus, $\mathcal{X}$ is a preferential structure iff $\eta$ is the identity.

See Diagram 3.3

Diagram 3.3

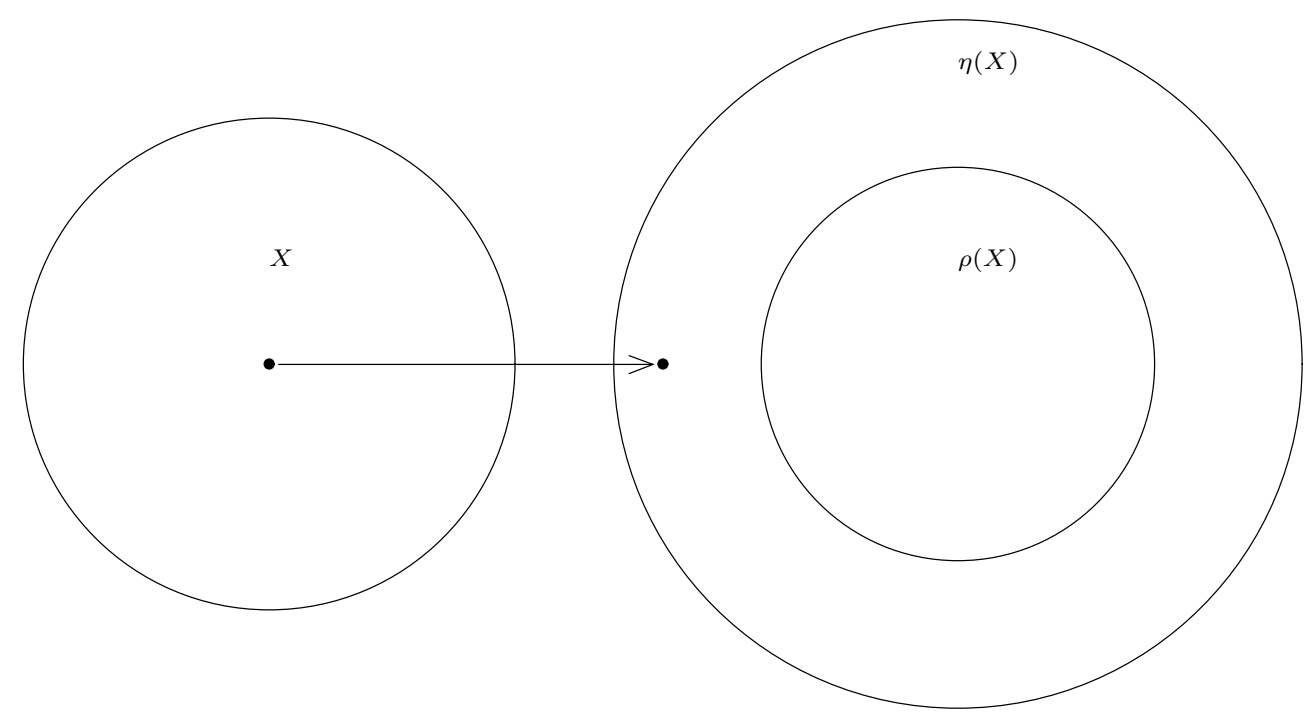

Attacking structure 
(Note that it does not seem very useful to generalize the notion of smoothness from preferential structures to general attacking structures, as, in the general case, the minimizing set $X$ and the result $\rho(X)$ may be disjoint.)

The following result is the first positive representation result of this paper, and shows that we can obtain (almost) anything with level 2 structures.

\section{Proposition 3.7}

Let $\eta, \rho: \mathcal{Y} \rightarrow \mathcal{P}(U)$. Then there is an attacking level 2 structure relative to $\eta$ representing $\rho$ iff

(1) $\rho(X) \subseteq \eta(X)$ for all $X \in \mathcal{Y}$,

(2) $\rho(\emptyset)=\eta(\emptyset)$ if $\emptyset \in \mathcal{Y}$.

(2) is, of course, void for preferential structures.

\section{Proof:}

(A) The construction

We make a two stage construction.

(A.1) Stage 1.

In stage one, consider (almost as usual)

$\mathcal{U}:=<\mathcal{X},\left\{\alpha_{i}: i \in I\right\}>$ where

$\mathcal{X}:=\{\langle x, f>: x \in U, f \in \Pi\{X \in \mathcal{Y}: x \in \eta(X)-\rho(X)\}\}$,

$\alpha: x^{\prime} \rightarrow<x, f>: \Leftrightarrow x^{\prime} \in \operatorname{ran}(f)$. Attention: $x^{\prime} \in X$, not $x^{\prime} \in \rho(X)$ !

(A.2) Stage 2.

Let $\mathcal{X}^{\prime}$ be the set of all $<x, f, X>$ s.t. $\langle x, f>\in \mathcal{X}$ and

(a) either $X$ is some dummy value, say *

or

(b) all of the following (1) - (4) hold:

(1) $X \in \mathcal{Y}$,

(2) $x \in \rho(X)$,

(3) there is $X^{\prime} \subseteq X, x \in \eta\left(X^{\prime}\right)-\rho\left(X^{\prime}\right), X^{\prime} \in \mathcal{Y}$, (thus $\operatorname{ran}(f) \cap X \neq \emptyset$ by definition),

(4) $\forall X^{\prime \prime} \in \mathcal{Y} .\left(X \subseteq X^{\prime \prime}, x \in \eta\left(X^{\prime \prime}\right)-\rho\left(X^{\prime \prime}\right) \rightarrow\left(\operatorname{ran}(f) \cap X^{\prime \prime}\right)-X \neq \emptyset\right)$.

(Thus, $f$ chooses in (4) for $X^{\prime \prime}$ also outside $X$. If there is no such $X^{\prime \prime}$, (4) is void, and only (1) - (3) need to hold, i.e. we may take any $f$ with $<x, f>\in \mathcal{X}$.)

See Diagram 3.4 . 


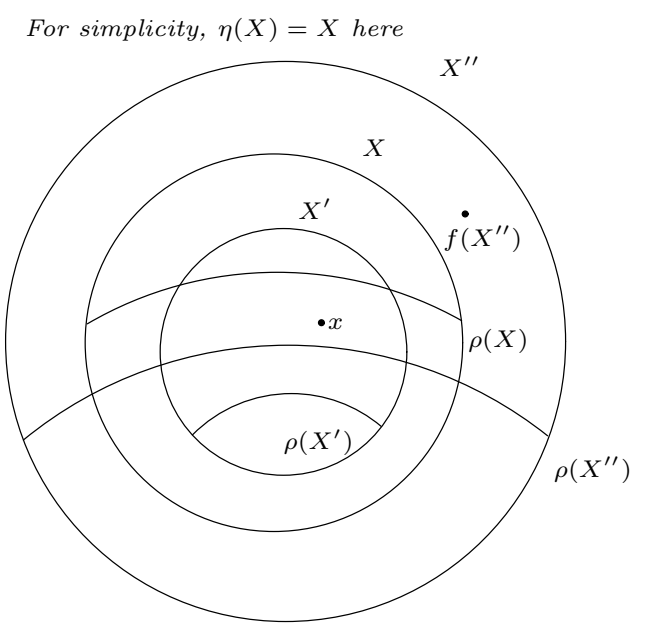

\section{Diagram 3.4 The complicated case}

Note: If (1) - (3) are satisfied for $x$ and $X$, then we will find $f$ s.t. $\langle x, f>\in \mathcal{X}$, and $\langle x, f, X>$ satisfies $(1)-(4):$ As $X \nRightarrow X^{\prime \prime}$ for $X^{\prime \prime}$ as in (4), we find $f$ which chooses for such $X^{\prime \prime}$ outside of $X$.

So for any $\langle x, f\rangle \in \mathcal{X}$, there is $\langle x, f, *\rangle$, and maybe also some $\left\langle x, f, X>\right.$ in $\mathcal{X}^{\prime}$.

Let again for any $x^{\prime},<x, f, X>\in \mathcal{X}^{\prime}$

$\alpha: x^{\prime} \rightarrow<x, f, X>: \Leftrightarrow x^{\prime} \in \operatorname{ran}(f)$

(A.3) Adding arrows.

Consider $x^{\prime}$ and $\langle x, f, X\rangle$.

If $X=*$, or $x^{\prime} \notin X$, we do nothing, i.e. leave a simple arrow $\alpha: x^{\prime} \rightarrow<x, f, X>\Leftrightarrow x^{\prime} \in \operatorname{ran}(f)$.

If $X \in \mathcal{Y}$, and $x^{\prime} \in X$, and $x^{\prime} \in \operatorname{ran}(f)$, we make $X$ many copies of the attacking arrow and have then: $<\alpha, x^{\prime \prime}>: x^{\prime} \rightarrow<x, f, X>$ for all $x^{\prime \prime} \in X$.

In addition, we add attacks on the $\left\langle\alpha, x^{\prime \prime}>:<\beta, x^{\prime \prime}>: x^{\prime \prime} \rightarrow<\alpha, x^{\prime \prime}>\right.$ for all $x^{\prime \prime} \in X$.

The full structure $\mathcal{Z}$ is thus:

$\mathcal{X}^{\prime}$ is the set of elements.

If $x^{\prime} \in \operatorname{ran}(f)$, and $X=*$ or $x^{\prime} \notin X$ then $\alpha: x^{\prime} \rightarrow<x, f, X>$

if $x^{\prime} \in \operatorname{ran}(f)$, and $X \neq *$ and $x^{\prime} \in X$ then

(a) $<\alpha, x^{\prime \prime}>$ : $x^{\prime} \rightarrow<x, f, X>$ for all $x^{\prime \prime} \in X$,

(b) $<\beta, x^{\prime \prime}>: x^{\prime \prime} \rightarrow<\alpha, x^{\prime \prime}>$ for all $x^{\prime \prime} \in X$.

See Diagram 3.5 .

Diagram 3.5 


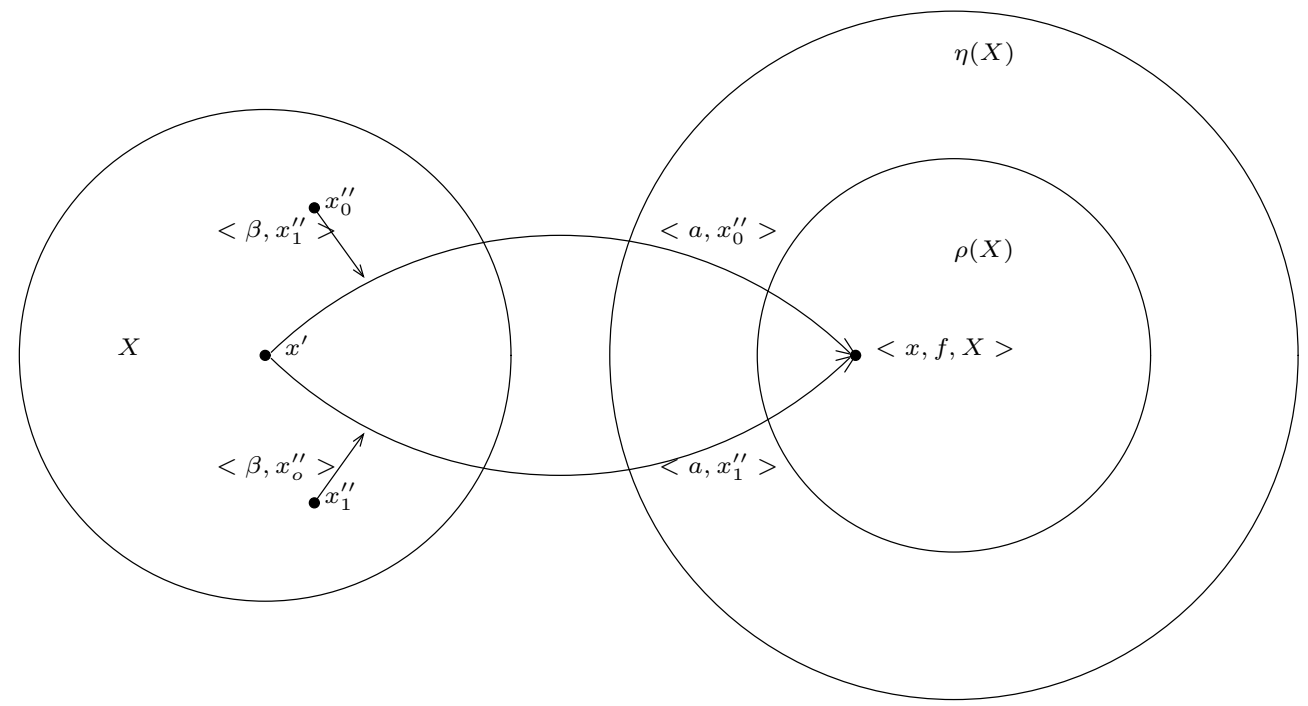

Attacking structure

(B) Representation

We have to show that this structure represents $\rho$ relative to $\eta$.

Let $y \in \eta(Y), Y \in \mathcal{Y}$.

Case 1. $y \in \rho(Y)$.

We have to show that there is $\left\langle y, g, Y^{\prime \prime}>\right.$ s.t. there is no valid $\alpha: y^{\prime} \rightarrow<y, g, Y^{\prime \prime}>, y^{\prime} \in Y$. In Case 1.1 below, $Y^{\prime \prime}$ will be $*$, in Case $1.2, Y^{\prime \prime}$ will be $Y, g$ will be chosen suitably.

Case 1.1. There is no $Y^{\prime} \subseteq Y, y \in \eta\left(Y^{\prime}\right)-\rho\left(Y^{\prime}\right), Y^{\prime} \in \mathcal{Y}$.

So for all $Y^{\prime}$ with $y \in \eta\left(Y^{\prime}\right)-\rho\left(Y^{\prime}\right) Y^{\prime}-Y \neq \emptyset$. Let $g \in \Pi\left\{Y^{\prime}-Y: y \in \eta\left(Y^{\prime}\right)-\rho\left(Y^{\prime}\right)\right\}$. Then $\operatorname{ran}(g) \cap Y=\emptyset$, and $\langle y, g, *\rangle$ is not attacked from $Y$. $(<y, g\rangle$ was already not attacked in $\mathcal{X}$.)

Case 1.2. There is $Y^{\prime} \subseteq Y, y \in \eta\left(Y^{\prime}\right)-\rho\left(Y^{\prime}\right), Y^{\prime} \in \mathcal{Y}$.

Let now $\left\langle y, g, Y>\in \mathcal{X}^{\prime}\right.$, s.t. $g\left(Y^{\prime \prime}\right) \notin Y$ if $Y \subseteq Y^{\prime \prime}, y \in \eta\left(Y^{\prime \prime}\right)-\rho\left(Y^{\prime \prime}\right), Y^{\prime \prime} \in \mathcal{Y}$. As noted above, such $g$ and thus $\langle y, g, Y>$ exist. Fix $\langle y, g, Y>$.

Consider any $y^{\prime} \in \operatorname{ran}(g)$. If $y^{\prime} \notin Y, y^{\prime}$ does not attack $\left\langle y, g, Y>\right.$ in $Y$. Suppose $y^{\prime} \in Y$. We had made $Y$ many copies $\left\langle\alpha, y^{\prime \prime}\right\rangle, y^{\prime \prime} \in Y$ with $\left.\left\langle\alpha, y^{\prime \prime}\right\rangle: y^{\prime} \rightarrow<y, g, Y\right\rangle$ and had added the level 2 arrows $<\beta, y^{\prime \prime}>: y^{\prime \prime} \rightarrow<\alpha, y^{\prime \prime}>$ for $y^{\prime \prime} \in Y$. So all copies $<\alpha, y^{\prime \prime}>$ are destroyed in $Y$. This was done for all $y^{\prime} \in Y, y^{\prime} \in \operatorname{ran}(g)$, so $\langle y, g, Y\rangle$ is now not (validly) attacked in $Y$ any more.

Case 2. $y \in \eta(Y)-\rho(Y)$.

Let $\left\langle y, g, Y^{\prime}\right\rangle$ (where $Y^{\prime}$ can be $*$ ) be any copy of $y$, we have to show that there is $z \in Y, \alpha: z \rightarrow<$ $y, g, Y^{\prime}>$, or some $\left\langle\alpha, z^{\prime}>: z \rightarrow<y, g, Y^{\prime}>, z^{\prime} \in Y^{\prime}\right.$, which is not destroyed by some level 2 arrow $<\beta, z^{\prime}>: z^{\prime} \rightarrow<\alpha, z^{\prime}>, z^{\prime} \in Y$. 
As $y \in \eta(Y)-\rho(Y), \operatorname{ran}(g) \cap Y \neq \emptyset$, so there is $z \in \operatorname{ran}(g) \cap Y$. Fix such $z$. (We will modify the choice of $z$ only in Case 2.2.2 below.)

Case 2.1. $Y^{\prime}=*$.

As $z \in \operatorname{ran}(g), \alpha: z \rightarrow<y, g, *>$. (There were no level 2 arrows introduced for this copy.)

Case 2.2. $Y^{\prime} \neq *$.

So $<y, g, Y^{\prime}>$ satisfies the conditions (1) - (4) of (b) at the beginning of the proof.

If $z \notin Y^{\prime}$, we are done, as $\alpha: z \rightarrow<y, g, Y^{\prime}>$, and there were no level 2 arrows introduced in this case. If $z \in Y^{\prime}$, we had made $Y^{\prime}$ many copies $\left.\left\langle\alpha, z^{\prime}\right\rangle,\left\langle\alpha, z^{\prime}\right\rangle: z \rightarrow<y, g, Y^{\prime}\right\rangle$, one for each $z^{\prime} \in Y^{\prime}$. Each $<\alpha, z^{\prime}>$ was destroyed by $\left\langle\beta, z^{\prime}>: z^{\prime} \rightarrow<\alpha, z^{\prime}>, z^{\prime} \in Y^{\prime}\right.$.

Case 2.2.1. $Y^{\prime} \nsubseteq Y$.

Let $z^{\prime \prime} \in Y^{\prime}-\mathrm{Y}$, then $\left\langle\alpha, z^{\prime \prime}>: z \rightarrow<y, g, Y^{\prime}>\right.$ is destroyed only by $\left\langle\beta, z^{\prime \prime}>: z^{\prime \prime} \rightarrow<\alpha, z^{\prime \prime}>\right.$ in $Y^{\prime}$, but not in $Y$, as $z^{\prime \prime} \notin Y$, so $\left\langle y, g, Y^{\prime}\right\rangle$ is attacked by $\left\langle\alpha, z^{\prime \prime}>: z \rightarrow\left\langle y, g, Y^{\prime}\right\rangle\right.$, valid in $Y$.

Case 2.2.2. $Y^{\prime} \varsubsetneqq Y\left(Y=Y^{\prime}\right.$ is impossible, as $\left.y \in \rho\left(Y^{\prime}\right), y \notin \rho(Y)\right)$.

Then there was by definition (condition (b) (4)) some $z^{\prime} \in(\operatorname{ran}(g) \cap Y)-Y^{\prime}$ and $\left.\alpha: z^{\prime} \rightarrow<y, g, Y^{\prime}\right\rangle$ is valid, as $z^{\prime} \notin Y^{\prime}$. (In this case, there are no copies of $\alpha$ and no level 2 arrows.)

\section{Corollary 3.8}

(1) We cannot distinguish general structures of level 2 from those of higher levels by their $\rho$-functions relative to $\eta$.

(2) Let $U$ be the universe, $\mathcal{Y} \subseteq \mathcal{P}(U), \mu: \mathcal{Y} \rightarrow \mathcal{P}(U)$. Then any $\mu$ satisfying $(\mu \subseteq)$ can be represented by a level 2 preferential structure. (Choose $\eta=$ identity.)

Again, we cannot distinguish general structures of level 2 from those of higher levels by their $\mu$-functions.

A remark on the function $\eta$ :

We can also obtain the function $\eta$ via arrows. Of course, then we need positive arrows (not only negative arrows against negative arrows, as we first need to have something positive).

If $\eta$ is the identity, we can make a positive arrow from each point to itself. Otherwise, we can connect every point to every point by a positive arrow, and then choose those we really want in $\eta$ by a choice function obtained from arrows just as we obtained $\rho$ from arrows.

\subsubsection{Results on total smoothness}

\section{Fact 3.9}


Let $X, Y \in \mathcal{Y}, \mathcal{X}$ a level $n$ structure. Let $\langle\alpha, k>:<x, i>\rightarrow<y, j>$, where $\langle y, j>$ may itself be (a copy of) an arrow.

(1) Let $n>1, X \subseteq Y,\langle\alpha, k>\in X$ a level $n-1$ arrow in $\mathcal{X}\lceil X$. If $\langle\alpha, k\rangle$ is valid in $\mathcal{X}\lceil Y$, then it is valid in $\mathcal{X}\lceil X$.

(2) Let $\mathcal{X}$ be totally smooth, $\mu(X) \subseteq Y, \mu(Y) \subseteq X,\langle\alpha, k>\in \mathcal{X}\lceil X \cap Y$, then $\langle\alpha, k>$ is valid in $\mathcal{X}\lceil X$ iff it is valid in $\mathcal{X}\lceil Y$.

Note that we will also sometimes write $X$ for $\mathcal{X}\lceil X$, when the context is clear.

\section{Proof:}

(1) If $\langle\alpha, k\rangle$ is not valid in $\mathcal{X}\lceil X$, then there must be a level $n$ arrow $\langle\beta, r\rangle:\langle z, s\rangle \rightarrow\langle\alpha, k\rangle$ in $\mathcal{X}\lceil X \subseteq \mathcal{X}\lceil Y .<\beta, r>$ must be valid in $\mathcal{X}\lceil X$ and $\mathcal{X}\lceil Y$, as there are no level $n+1$ arrows. So $\langle\alpha, k>$ is not valid in $\mathcal{X}\lceil Y$, contradiction.

(2) By downward induction. Case $n:\langle\alpha, k>\in \mathcal{X}\lceil X \cap Y$, so it is valid in both as there are no level $n+1$ arrows. Case $m \rightarrow m-1$ : Let $\langle\alpha, k>\in \mathcal{X}\lceil X \cap Y$ be a level $m-1$ arrow valid in $\mathcal{X}\lceil X$, but not in $\mathcal{X}\lceil Y$. So there must be a level $m$ arrow $\langle\beta, r>:\langle z, s\rangle \rightarrow<\alpha, k>$ valid in $\mathcal{X}\lceil Y$. By total smoothness, we may assume $z \in \mu(Y) \subseteq X$, so $\langle\beta, r>\in \mathcal{X}\lceil X$ valid by induction hypothesis. So $\langle\alpha, k>$ is not valid in $\mathcal{X}\lceil X$, contradiction.

\section{Corollary 3.10}

Let $X, Y \in \mathcal{Y}, \mathcal{X}$ a totally smooth level $n$ structure, $\mu(X) \subseteq Y, \mu(Y) \subseteq X$. Then $\mu(X)=\mu(Y)$.

\section{Proof:}

Let $x \in \mu(X)-\mu(Y)$. Then by $\mu(X) \subseteq Y, x \in Y$, so there must be for all $<x, i>\in \mathcal{X}$ an arrow $\langle\alpha, k\rangle:\langle y, j\rangle \rightarrow\langle x, i>$ valid in $\mathcal{X}\lceil Y$, wlog. $y \in \mu(Y) \subseteq X$ by total smoothness. So by Fact 3.9, (2), $\langle\alpha, k\rangle$ is valid in $\mathcal{X}\lceil X$. This holds for all $\langle x, i\rangle$, so $x \notin \mu(X)$, contradiction.

\section{Fact 3.11}

There are situations satisfying $(\mu \subseteq)+(\mu C U M)+(\cap)$ which cannot be represented by level 2 totally smooth preferential structures.

The proof is given in the following example.

\section{Example 3.6}

Let $Y:=\left\{x, y, y^{\prime}\right\}, X:=\{x, y\}, X^{\prime}:=\left\{x, y^{\prime}\right\}$. Let $\mathcal{Y}:=\mathcal{P}(Y)$. Let $\mu(Y):=\left\{y, y^{\prime}\right\}, \mu(X):=\mu\left(X^{\prime}\right):=$ $\{x\}$, and $\mu(Z):=Z$ for all other sets.

Obviously, this satisfies $(\cap),(\mu \subseteq)$, and $(\mu C U M)$.

Suppose $\mathcal{X}$ is a totally smooth level 2 structure representing $\mu$.

So $\mu(X)=\mu\left(X^{\prime}\right) \subseteq Y-\mu(Y), \mu(Y) \subseteq X \cup X^{\prime}$. Let $<x, i>$ be minimal in $\mathcal{X}\lceil X$. As $<x, i>$ cannot be minimal in $\mathcal{X}\lceil Y$, there must be $\alpha:<z, j>\rightarrow<x, i>$, valid in $\mathcal{X}\lceil Y$. 
Case 1: $z \in X^{\prime}$.

So $\alpha \in \mathcal{X}\left\lceil X^{\prime}\right.$. If $\alpha$ is valid in $\mathcal{X}\left\lceil X^{\prime}\right.$, there must be $\alpha^{\prime}:<x^{\prime}, i^{\prime}>\rightarrow<x, i>, x^{\prime} \in \mu\left(X^{\prime}\right)$, valid in $\mathcal{X}\left\lceil X^{\prime}\right.$, and thus in $\mathcal{X}\left\lceil X\right.$, by $\mu(X)=\mu\left(X^{\prime}\right)$ and Fact 3.9 (2). This is impossible, so there must be $\beta:\left\langle x^{\prime}, i^{\prime}\right\rangle \rightarrow \alpha$, $x^{\prime} \in \mu\left(X^{\prime}\right)$, valid in $\mathcal{X}\left\lceil X^{\prime}\right.$. As $\beta$ is in $\mathcal{X}\lceil Y$ and $\mathcal{X}$ a level $\leq 2$ structure, $\beta$ is valid in $\mathcal{X}\lceil Y$, so $\alpha$ is not valid in $\mathcal{X}\lceil Y$, contradiction.

Case 2: $z \in X$.

$\alpha$ cannot be valid in $\mathcal{X}\left\lceil X\right.$, so there must be $\beta:<x^{\prime}, i^{\prime}>\rightarrow \alpha, x^{\prime} \in \mu(X)$, valid in $\mathcal{X}\lceil X$. Again, as $\beta$ is in $\mathcal{X}\lceil Y$ and $\mathcal{X}$ a level $\leq 2$ structure, $\beta$ is valid in $\mathcal{X}\lceil Y$, so $\alpha$ is not valid in $\mathcal{X}\lceil Y$, contradiction.

It is unknown to the authors whether an analogon is true for essential smoothness, i.e. whether there are examples of such $\mu$ function which need at least level 3 essentially smooth structures for representation. Proposition 3.15 below shows that such structures suffice, but we do not know whether level 3 is necessary.

\section{Fact 3.12}

Above Example 3.6 can be solved by a totally smooth level 3 structure:

Let $\alpha_{1}: x \rightarrow y, \alpha_{2}: x \rightarrow y^{\prime}, \alpha_{3}: y \rightarrow x, \beta_{1}: y \rightarrow \alpha_{2}, \beta_{2}: y^{\prime} \rightarrow \alpha_{1}, \beta_{3}: y \rightarrow \alpha_{3}, \beta_{4}: x \rightarrow \alpha_{3}, \gamma_{1}: y^{\prime} \rightarrow \beta_{3}$, $\gamma_{2}: y^{\prime} \rightarrow \beta_{4}$.

See Diagram 3.6.

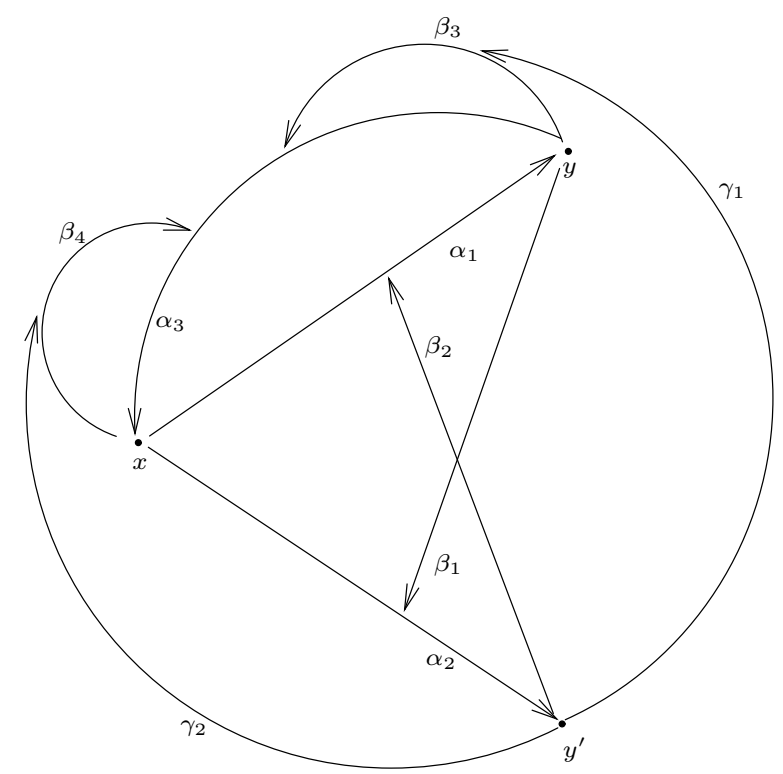

Diagram 3.6 Solution by smooth level 3 structure

The legal subdiagram generated by $X$ contains $\alpha_{1}, \alpha_{3}, \beta_{3}, \beta_{4} . \alpha_{1}, \beta_{3}, \beta_{4}$ are valid, so $\mu(X)=\{x\}$. 
The legal subdiagram generated by $X^{\prime}$ contains $\alpha_{2} . \alpha_{2}$ is valid, so $\mu\left(X^{\prime}\right)=\{x\}$.

In the full diagram, $\alpha_{3}, \beta_{1}, \beta_{2}, \gamma_{1}, \gamma_{2}$ are valid, so $\mu(Y)=\left\{y, y^{\prime}\right\}$.

\section{Remark 3.13}

Example 3.7 together with Corollary 3.10 show that $(\mu \subseteq)$ and $(\mu C U M)$ without $(\cap)$ do not guarantee representability by a level $n$ totally smooth structure.

\section{Example 3.7}

We show here $(\mu \subseteq)+(\mu C U M) \nRightarrow(\mu \subseteq \supseteq)$.

Consider $X:=\{a, b, c\}, Y:=\{a, b, d\}, f(X):=\{a\}, f(Y):=\{a, b\}, \mathcal{Y}:=\{X, Y\}$. (If $f(\{a, b\})$ were defined, we would have $f(X)=f(\{a, b\})=f(Y)$, contradiction. $)$

Obviously, $(\mu \subseteq)$ and $(\mu C U M)$ hold, but not $(\mu \subseteq \supseteq)$.

\subsubsection{Results on essential smoothness}

\section{Definition 3.17}

Let $\mu: \mathcal{Y} \rightarrow \mathcal{P}(U)$ and $\mathcal{X}$ be given, let $\alpha:<y, j>\rightarrow<x, i>\in \mathcal{X}$.

Define

$\boldsymbol{O}(\alpha):=\{Y \in \mathcal{Y}: x \in Y-\mu(Y), y \in \mu(Y)\}$,

$\boldsymbol{D}(\alpha):=\{X \in \mathcal{Y}: x \in \mu(X), y \in X\}$,

$\Pi(\boldsymbol{O}, \alpha):=\Pi\{\mu(Y): Y \in \boldsymbol{O}(\alpha)\}$,

$\Pi(\boldsymbol{D}, \alpha):=\Pi\{\mu(X): X \in \boldsymbol{D}(\alpha)\}$.

\section{Lemma 3.14}

Let $U$ be the universe, $\mu: \mathcal{Y} \rightarrow \mathcal{P}(U)$. Let $\mu$ satisfy $(\mu \subseteq)+(\mu \subseteq \supseteq)$.

Let $\mathcal{X}$ be a level 1 preferential structure, $\alpha:\langle y, j>\rightarrow<x, i>, \boldsymbol{O}(\alpha) \neq \emptyset, \boldsymbol{D}(\alpha) \neq \emptyset$.

We can modify $\mathcal{X}$ to a level 3 structure $\mathcal{X}^{\prime}$ by introducing level 2 and level 3 arrows s.t. no copy of $\alpha$ is valid in any $X \in \boldsymbol{D}(\alpha)$, and in every $Y \in \boldsymbol{O}(\alpha)$ at least one copy of $\alpha$ is valid. (More precisely, we should write $\mathcal{X}^{\prime}\lceil X$ etc.)

Thus, in $\mathcal{X}^{\prime}$,

(1) $<x, i>$ will not be minimal in any $Y \in \boldsymbol{O}(\alpha)$,

(2) if $\alpha$ is the only arrow minimizing $\langle x, i>$ in $X \in \boldsymbol{D}(\alpha),\langle x, i\rangle$ will now be minimal in $X$. 
The construction is made independently for all such arrows $\alpha \in \mathcal{X}$.

(This is probably the main technical result of the paper.)

\section{Proof:}

(1) The construction

Make $\Pi(\boldsymbol{D}, \alpha)$ many copies of $\alpha:\{\langle\alpha, f>: f \in \Pi(\boldsymbol{D}, \alpha)\}$, all $\langle\alpha, f\rangle:\langle y, j\rangle \rightarrow\langle x, i\rangle$. Note that $<\alpha, f>\in X$ for all $X \in \boldsymbol{D}(\alpha)$ and $<\alpha, f>\in Y$ for all $Y \in \boldsymbol{O}(\alpha)$.

Add to the structure $<\beta, f, X_{r}, g>:<f\left(X_{r}\right), i_{r}>\rightarrow<\alpha, f>$, for any $X_{r} \in \boldsymbol{D}(\alpha)$, and $g \in \Pi(\boldsymbol{O}, \alpha)($ and some or all $i_{r}$ - this does not matter).

For all $Y_{s} \in \boldsymbol{O}(\alpha)$ :

if $\mu\left(Y_{s}\right) \nsubseteq X_{r}$ and $f\left(X_{r}\right) \in Y_{s}$, then add to the structure $<\gamma, f, X_{r}, g, Y_{s}>:<g\left(Y_{s}\right), j_{s}>\rightarrow<\beta, f, X_{r}, g>$ (again for all or some $j_{s}$ ),

if $\mu\left(Y_{s}\right) \subseteq X_{r}$ or $f\left(X_{r}\right) \notin Y_{s},<\gamma, f, X_{r}, g, Y_{s}>$ is not added.

See Diagram 3.7. 


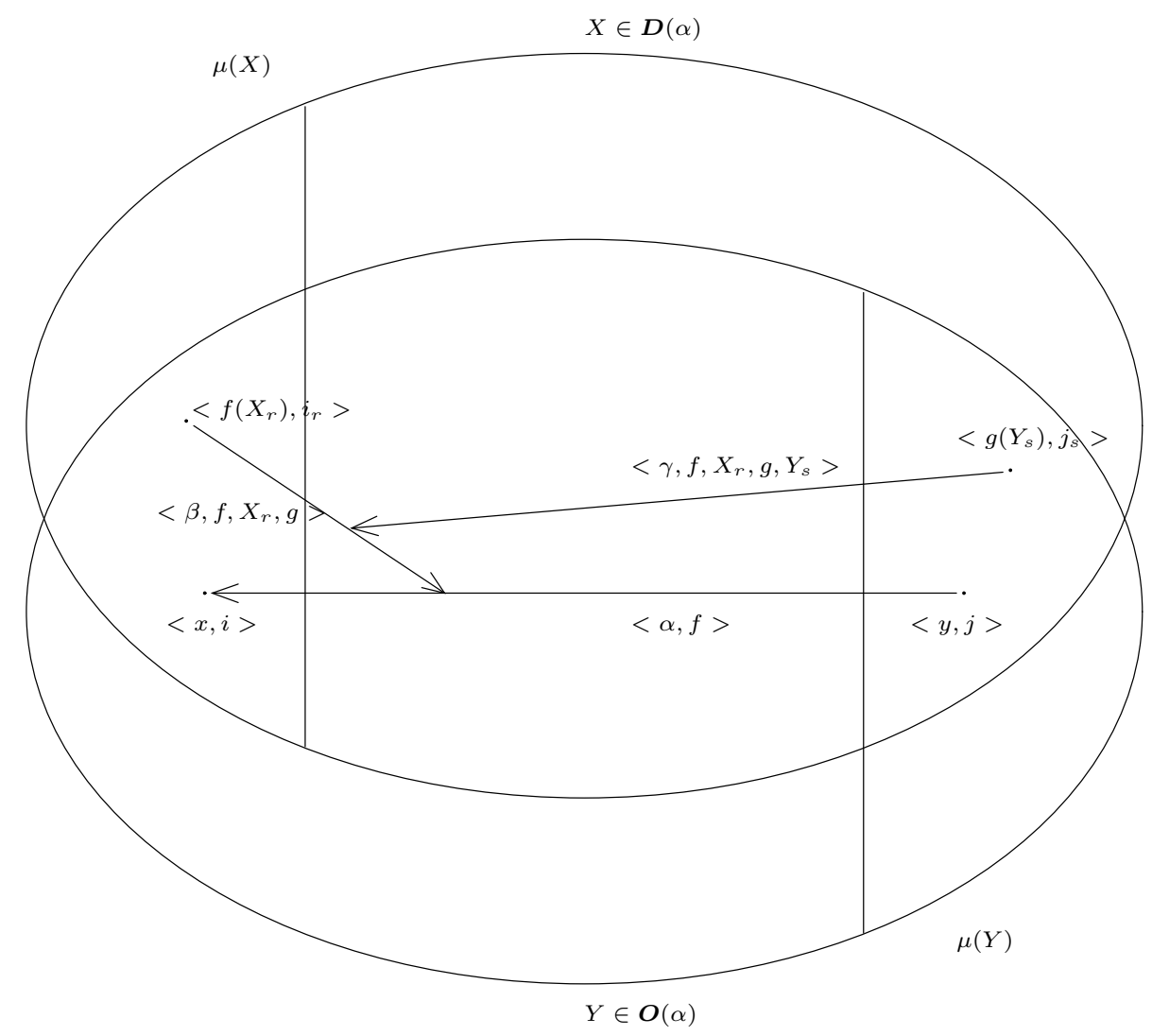

The construction

\section{Diagram 3.7}

$(2)$

Let $X_{r} \in \boldsymbol{D}(\alpha)$. We have to show that no $\langle\alpha, f\rangle$ is valid in $X_{r}$. Fix $f$.

$\langle\alpha, f\rangle$ is in $X_{r}$, so we have to show that for at least one $\left.g \in \Pi(\boldsymbol{O}, \alpha)<\beta, f, X_{r}, g\right\rangle$ is valid in $X_{r}$, i.e. that for this $g$, no $<\gamma, f, X_{r}, g, Y_{s}>:<g\left(Y_{s}\right), j_{s}>\rightarrow<\beta, f, X_{r}, g>, Y_{s} \in \boldsymbol{O}(\alpha)$ attacks $<\beta, f, X_{r}, g>$ in $X_{r}$.

We define $g$. Take $Y_{s} \in \boldsymbol{O}(\alpha)$.

Case 1: $\mu\left(Y_{s}\right) \subseteq X_{r}$ or $f\left(X_{r}\right) \notin Y_{s}$ : choose arbitrary $g\left(Y_{s}\right) \in \mu\left(Y_{s}\right)$.

Case 2: $\mu\left(Y_{s}\right) \nsubseteq X_{r}$ and $f\left(X_{r}\right) \in Y_{s}$ : Choose $g\left(Y_{s}\right) \in \mu\left(Y_{s}\right)-X_{r}$.

In Case $1,<\gamma, f, X_{r}, g, Y_{s}>$ does not exist, so it cannot attack $\left\langle\beta, f, X_{r}, g\right\rangle$. 
In Case $2,<\gamma, f, X_{r}, g, Y_{s}>:<g\left(Y_{s}\right), j_{s}>\rightarrow<\beta, f, X_{r}, g>$ is not in $X_{r}$, as $g\left(Y_{s}\right) \notin X_{r}$.

Thus, no $\left\langle\gamma, f, X_{r}, g, Y_{s}>:<g\left(Y_{s}\right), j_{s}>\rightarrow<\beta, f, X_{r}, g>, Y_{s} \in \boldsymbol{O}(\alpha)\right.$ attacks $\left\langle\beta, f, X_{r}, g>\right.$ in $X_{r}$.

So $\forall<\alpha, f>:<y, j>\rightarrow<x, i>$

$$
\begin{aligned}
& y \in X_{r} \rightarrow \exists<\beta, f, X_{r}, g>:<f\left(X_{r}\right), i_{r}>\rightarrow<\alpha, f> \\
&\left(f\left(X_{r}\right) \in \mu\left(X_{r}\right) \wedge \neg \exists<\gamma, f, X_{r}, g, Y_{s}>:<g\left(Y_{s}\right), j_{s}>\rightarrow<\beta, f, X_{r}, g>. g\left(Y_{s}\right) \in X_{r}\right) .
\end{aligned}
$$

But $\left\langle\beta, f, X_{r}, g\right\rangle$ was constructed only for $\langle\alpha, f\rangle$, so was $\left\langle\gamma, f, X_{r}, g, Y_{s}\right\rangle$, and there was no other $<\gamma, i>$ attacking $<\beta, f, X_{r}, g>$, so we are done.

(3)

Let $Y_{s} \in \boldsymbol{O}(\alpha)$. We have to show that at least one $\langle\alpha, f\rangle$ is valid in $Y_{s}$.

We define $f \in \Pi(\boldsymbol{D}, \alpha)$. Take $X_{r}$.

If $\mu\left(X_{r}\right) \nsubseteq Y_{s}$, choose $f\left(X_{r}\right) \in \mu\left(X_{r}\right)-Y_{s}$. If $\mu\left(X_{r}\right) \subseteq Y_{s}$, choose arbitrary $f\left(X_{r}\right) \in \mu\left(X_{r}\right)$.

All attacks on $\left\langle x, f>\right.$ have the form $\left\langle\beta, f, X_{r}, g>:<f\left(X_{r}\right), i_{r}>\rightarrow<\alpha, f>, X_{r} \in \boldsymbol{D}(\alpha), g \in \Pi(\boldsymbol{O}, \alpha)\right.$.

We have to show that they are either not in $Y_{s}$, or that they are themselves attacked in $Y_{s}$.

Case 1: $\mu\left(X_{r}\right) \nsubseteq Y_{s}$. Then $f\left(X_{r}\right) \notin Y_{s}$, so $\left.<\beta, f, X_{r}, g\right\rangle:<f\left(X_{r}\right), i_{r}>\rightarrow<\alpha, f>$ is not in $Y_{s}$ (for no $g)$.

Case 2: $\mu\left(X_{r}\right) \subseteq Y_{s}$. Then $\mu\left(Y_{s}\right) \nsubseteq X_{r}$ by $(\mu \subseteq \supseteq)$ and $f\left(X_{r}\right) \in Y_{s}$, so $<\beta, f, X_{r}, g>:<f\left(X_{r}\right), i_{r}>\rightarrow<$ $\alpha, f>$ is in $Y_{s}$ (for all $g$ ). Take any $g \in \Pi(\boldsymbol{O}, \alpha)$. As $\mu\left(Y_{s}\right) \nsubseteq X_{r}$ and $f\left(X_{r}\right) \in Y_{s},<\gamma, f, X_{r}, g, Y_{s}>:<$ $g\left(Y_{s}\right), j_{s}>\rightarrow<\beta, f, X_{r}, g>$ is defined, and $g\left(Y_{s}\right) \in \mu\left(Y_{s}\right)$, so it is in $Y_{s}$ (for all $g$ ). Thus, $\left\langle\beta, f, X_{r}, g\right\rangle$ is attacked in $Y_{s}$.

Thus, for this $f$, all $\left\langle\beta, f, X_{r}, g\right\rangle$ are either not in $Y_{s}$, or attacked in $Y_{s}$, thus for this $f,\langle\alpha, f\rangle$ is valid in $Y_{s}$.

So for this $<x, i>$

$$
\begin{aligned}
& \exists<\alpha, f>:<y, j>\rightarrow<x, i>. y \in \mu\left(Y_{s}\right) \wedge \\
& \text { (a) } \neg \exists<\beta, f, X_{r}, g>:<f\left(X_{r}\right), i>\rightarrow<\alpha, f>. f\left(X_{r}\right) \in Y_{s} \\
& \text { or } \\
& \begin{aligned}
\text { (b) } \forall<\beta, f, X_{r}, g>:<f\left(X_{r}\right), i>\rightarrow<\alpha, f> \\
\quad\left(f\left(X_{r}\right) \in Y_{s} \rightarrow\right. \\
\left.\quad \exists<\gamma, f, X_{r}, g, Y_{s}>:<g\left(Y_{s}\right), j_{s}>\rightarrow<\beta, f, X_{r}, g>. g\left(Y_{s}\right) \in \mu\left(Y_{s}\right)\right) .
\end{aligned}
\end{aligned}
$$

As we made copies of $\alpha$ only, introduced only $\beta^{\prime}$ s attacking the $\alpha$-copies, and $\gamma^{\prime}$ s attacking the $\beta^{\prime}$ s, the construction is independent for different $\alpha^{\prime}$ s.

\section{Proposition 3.15}

Let $U$ be the universe, $\mu: \mathcal{Y} \rightarrow \mathcal{P}(U)$.

Then any $\mu$ satisfying $(\mu \subseteq),(\cap),(\mu C U M)$ (or, alternatively, $(\mu \subseteq)$ and $(\mu \subseteq \supseteq)$ ) can be represented by a level 3 essentially smooth structure.

\section{Proof:}


In stage one, consider as usual $\mathcal{U}:=<\mathcal{X},\left\{\alpha_{i}: i \in I\right\}>$ where $\mathcal{X}:=\{<x, f>: x \in U, f \in \Pi\{\mu(X)$ : $X \in \mathcal{Y}, x \in X-\mu(X)\}\}$, and set $\alpha:<x^{\prime}, f^{\prime}>\rightarrow<x, f>: \Leftrightarrow x^{\prime} \in \operatorname{ran}(f)$.

For stage two:

Any level 1 arrow $\alpha:\langle y, j>\rightarrow<x, i>$ was introduced in stage one by some $Y \in \mathcal{Y}$ s.t. $y \in \mu(Y)$, $x \in Y-\mu(Y)$. Do the construction of Lemma 3.14 for all level 1 arrows of $\mathcal{X}$ in parallel or successively.

We have to show that the resulting structure represents $\mu$ and is essentially smooth. (Level 3 is obvious.)

(1) Representation

Suppose $x \in Y-\mu(Y)$. Then there was in stage 1 for all $\langle x, i>$ some $\alpha:\langle y, j\rangle \rightarrow\langle x, i>, y \in \mu(Y)$. We examine the $y$.

If there is no $X$ s.t. $x \in \mu(X), y \in X$, then there were no $\beta^{\prime}$ s and $\gamma^{\prime}$ s introduced for this $\alpha:<y, j>\rightarrow<$ $x, i>$, so $\alpha$ is valid.

If there is $X$ s.t. $x \in \mu(X), y \in X$, consider $\alpha:\langle y, j>\rightarrow<x, i>$. So $X \in \boldsymbol{D}(\alpha), Y \in \boldsymbol{O}(\alpha)$, so we did the construction of Lemma 3.14, and by its result, $\langle x, i\rangle$ is not minimal in $Y$.

Thus, in both cases, $\langle x, i\rangle$ is successfully attacked in $Y$, and no $\langle x, i\rangle$ is a minimal element in $Y$.

Suppose $x \in \mu(X)$ (we change notation to conform to Lemma 3.14). Fix $\langle x, i\rangle$.

If there is no $\alpha:\langle y, j>\rightarrow<x, i>, y \in X$, then $\langle x, i>$ is minimal in $X$, and we are done.

If there is $\alpha$ or $\langle\alpha, k>:\langle y, j>\rightarrow\langle x, i>, y \in X$, then $\alpha$ originated from stage one through some $Y$ s.t. $x \in Y-\mu(Y)$, and $y \in \mu(Y)$. (Note that stage 2 of the construction did not introduce any new level 1 arrows - only copies of existing level 1 arrows.) So $X \in \boldsymbol{D}(\alpha), Y \in \boldsymbol{O}(\alpha)$, so we did the construction of Lemma 3.14, and by its result, $\langle x, i\rangle$ is minimal in $X$, and we are done again.

In both cases, all $\langle x, i\rangle$ are minimal elements in $X$.

(2) Essential smoothness. We have to show the conditions of Definition 3.12. We will, however, work with the reformulation given in Remark 3.5.

Case (1), $x \in \mu(X)$.

Case (1.1), there is $\langle x, i\rangle$ with no $\langle\alpha, f\rangle:\langle y, j\rangle \rightarrow\langle x, i\rangle, y \in X$. There is nothing to show.

Case (1.2), for all $\langle x, i\rangle$ there is $\langle\alpha, f\rangle:\langle y, j\rangle \rightarrow\langle x, i\rangle, y \in X$.

$\alpha$ was introduced in stage 1 by some $Y$ s.t. $x \in Y-\mu(Y), y \in X \cap \mu(Y)$, so $X \in \boldsymbol{D}(\alpha), Y \in \boldsymbol{O}(\alpha)$. In the proof of Lemma 3.14, at the end of (2), it was shown that

$$
\begin{aligned}
\exists<\beta, f, & X_{r}, g>:<f\left(X_{r}\right), i_{r}>\rightarrow<\alpha, f> \\
& \left(f\left(X_{r}\right) \in \mu\left(X_{r}\right) \wedge\right. \\
& \left.\neg \exists<\gamma, f, X_{r}, g, Y_{s}>:<g\left(Y_{s}\right), j_{s}>\rightarrow<\beta, f, X_{r}, g>g\left(Y_{s}\right) \in X_{r}\right) .
\end{aligned}
$$

By $f\left(X_{r}\right) \in \mu\left(X_{r}\right)$, condition (1) of Remark 3.5 is true.

Case (2), $x \notin \mu(Y)$. Fix $\langle x, i\rangle$. (We change notation back to $Y$.)

In stage 1, we constructed $\alpha:\langle y, j>\rightarrow<x, i>, y \in \mu(Y)$, so $Y \in \boldsymbol{O}(\alpha)$.

If $\boldsymbol{D}(\alpha)=\emptyset$, then there is no attack on $\alpha$, and the condition (2) of Remark 3.5 is trivially true.

If $\boldsymbol{D}(\alpha) \neq \emptyset$, we did the construction of Lemma 3.14, so

$\exists<\alpha, f>:<y, j>\rightarrow<x, i>. y \in \mu\left(Y_{s}\right) \wedge$

(a) $\neg \exists<\beta, f, X_{r}, g>:<f\left(X_{r}\right), i>\rightarrow<\alpha, f>. f\left(X_{r}\right) \in Y_{s}$

or 
(b) $\forall<\beta, f, X_{r}, g>:<f\left(X_{r}\right), i>\rightarrow<\alpha, f>$

$$
\begin{aligned}
& \left(f\left(X_{r}\right) \in Y_{s} \rightarrow\right. \\
& \exists<\gamma, f, X_{r}, g, Y_{s}>:<g\left(Y_{s}\right), j_{s}>\rightarrow<\beta, f, X_{r}, g>. g\left(Y_{s}\right) \in \mu\left(Y_{s}\right) .
\end{aligned}
$$

As the only attacks on $\left\langle\alpha, f>\right.$ had the form $\left\langle\beta, f, X_{r}, g\right\rangle$, and $g\left(Y_{s}\right) \in \mu\left(Y_{s}\right)$, condition (2) of Remark 3.5 is satisfied.

As said after Example 3.6, we do not know if level 3 is necessary for representation. We also do not know whether the same can be achieved with level 3, or higher, totally smooth structures.

\subsubsection{Translation to logics}

We turn to the translation to logics.

\section{Proposition 3.16}

Let $\sim$ be a logic for $\mathcal{L}$. Set $T^{\mathcal{M}}:=T h\left(\mu_{\mathcal{M}}(M(T))\right)$, where $\mathcal{M}$ is a generalized preferential structure, and $\mu_{\mathcal{M}}$ its choice function. Then

(1) there is a level 2 preferential structure $\mathcal{M}$ s.t. $\overline{\bar{T}}=T^{\mathcal{M}}$ iff (LLE), (CCL), (SC) hold for all $T, T^{\prime} \subseteq \mathcal{L}$.

(2) there is a level 3 essentially smooth preferential structure $\mathcal{M}$ s.t. $\overline{\bar{T}}=T^{\mathcal{M}}$ iff (LLE), (CCL), (SC), $(\subseteq \supseteq)$ hold for all $T, T^{\prime} \subseteq \mathcal{L}$.

The proof is an immediate consequence of Corollary 3.8 (2), Fact 3.6, Proposition 3.15, and Proposition 3.3 (10) and (11).

We leave aside the generalization of preferential structures to attacking structures relative to $\eta$, as this can cause problems, without giving real insight: It might well be that $\rho(X) \nsubseteq \eta(X)$, but, still, $\rho(X)$ and $\eta(X)$ might define the same theory - see Section 3.1.

\section{References}

[BS85] G.Bossu, P.Siegel, "Saturation, Nonmonotonic Reasoning and the Closed- World Assumption", Artificial Intelligence 25 (1985) 13-63

[Dun95] P.M.Dung, "On the acceptability of arguments and its fundamental role in nonmonotonic reasoning, logic programming and $n$-person games", Artificial Intelligence 77 (1995), p.321-357 
[Gab85] D.M.Gabbay, "Theoretical foundations for non-monotonic reasoning in expert systems". In: K.R.Apt (ed.), "Logics and Models of Concurrent Systems", Springer, Berlin, 1985, p.439-457

[KLM90] S.Kraus, D.Lehmann, M.Magidor, "Nonmonotonic reasoning, preferential models and cumulative logics", Artificial Intelligence, 44 (1-2), p.167-207, July 1990

[Sch04] K.Schlechta: "Coherent Systems", Elsevier, Amsterdam, 2004

[Sch06-t1] Karl Schlechta: "Domain closure conditions and definability preservation", HAL ccsd00084398, arXiv.org math.LO/0607189, submitted July 7, 2006

[Sch92] K.Schlechta: "Some results on classical preferential models", Journal of Logic and Computation, Oxford, Vol.2, No.6 (1992), p. 675-686

[Sho87b] Yoav Shoham: "A semantical approach to nonmonotonic logics". In Proc. Logics in Computer Science, p.275-279, Ithaca, N.Y., 1987, and In Proceed. IJCAI 87, p.388-392 